\title{
A COMMUTANT LIFTING THEOREM ON ANALYTIC POLYHEDRA
}

\author{
CALIN AMBROZIE \\ Institute of Mathematics, Romanian Academy \\ PO Box 1-764, 70700 Bucharest, Romania \\ E-mail: cambroz@imar.ro \\ JÖRG ESCHMEIER \\ Fachrichtung Mathematik, Universität des Saarlandes \\ Postfach 151150, D-66041 Saarbrücken, Germany \\ E-mail: eschmei@math.uni-sb.de
}

To Professor Wiestaw Żelazko on the occasion of his seventieth birthday

\begin{abstract}
In this note a commutant lifting theorem for vector-valued functional Hilbert spaces over generalized analytic polyhedra in $\mathbb{C}^{n}$ is proved. Let $T$ be the compression of the multiplication tuple $M_{z}$ to a $*$-invariant closed subspace of the underlying functional Hilbert space. Our main result characterizes those operators in the commutant of $T$ which possess a lifting to a multiplier with Schur class symbol. As an application we obtain interpolation results of NevanlinnaPick and Carathéodory-Fejér type for Schur class functions. Our methods apply in particular to the unit ball, the unit polydisc and the classical symmetric domains of types I, II and III.

In a paper from 1967 Sarason observed that commutant lifting results for contractions can be used to solve interpolation problems for bounded analytic functions on the unit disc. This idea was considerably extended in the work of Sz.-Nagy and Foiaş on Hilbert space contractions (see, for example, $[21,31]$ ). Each contraction $T \in B(H)$ of type $C_{.0}$ is, up to unitary equivalence, the compression of the multiplication operator with the independent variable $M_{z} \in B\left(H^{2}(\mathbb{D}, E)\right)$ on a vector-valued Hardy space to a closed linear subspace $M \subset H^{2}(\mathbb{D}, E)$ which is invariant for the adjoint $M_{z}^{*}$. A functional version of the commutant lifting theorem, suitable for applications to interpolation problems, says that each operator $X$ in the commutant of the compression $T=P_{M} M_{z} \mid M$ dilates to a
\end{abstract}

2000 Mathematics Subject Classification: Primary 47A57; Secondary 47A13, 47A20, 41A05.

The first-named author expresses his thanks to the Department of Mathematics of the University of Saarbrücken for its kind hospitality during the first stage of the preparation of the present work.

The paper is in final form and no version of it will be published elsewhere. 
multiplication operator $M_{f}$ given by a bounded analytic function $f \in H^{\infty}(\mathbb{D}, B(E))$ with $\|X\|=\|f\|_{\infty}$. Since the commutant of the multiplication operator $M_{z}$ on $H^{2}(\mathbb{D}, E)$ consists precisely of all multiplication operators $M_{f}$ with $f \in H^{\infty}(\mathbb{D}, B(E))$, the above result can be seen as the $C_{0^{0}}$-case of the abstract commutant lifting theorem for contractions.

If one replaces the Hardy space on the unit disc by the Hardy space on the open unit ball $\mathbb{B}$ in $\mathbb{C}^{n}$ and uses the commuting $n$-tuple $M_{z}=\left(M_{z_{1}}, \ldots, M_{z_{n}}\right) \in B\left(H^{2}(\mathbb{B}, E)\right)^{n}$ consisting of the multiplication operators with the coordinate functions instead of the operator $M_{z}$ on $H^{2}(\mathbb{D}, E)$, then the multivariable analogue of the above functional form of the commutant lifting theorem is no longer true. An easy way to show this, is to use the well-known fact that the ball version of the classical Nevanlinna-Pick theorem for functions in $H^{\infty}(\mathbb{B})$ is wrong (see e.g. [24]).

By von Neumann's inequality, the supremum norm of a bounded analytic function $f$ in $H^{\infty}(\mathbb{D}, B(E))$ can be described as

$$
\|f\|_{\infty}=\sup \{\|f(T)\| ; T \in B(H) \text { with }\|T\|<1\} .
$$

Here $H$ is a fixed separable, infinite-dimensional Hilbert space, and the operator $f(T)$ is formed with the help of an appropriate vector-valued analytic functional calculus which will be explained below.

If one substitutes $\mathbb{B}$ for $\mathbb{D}$ and replaces single contractions by the class of all $n$ contractions as introduced by Arveson in [8], then the space of all analytic functions $f \in \mathcal{O}(\mathbb{B}, B(E))$, for which the norm defined by the supremum in the above formula is finite, forms a contractively embedded Banach subalgebra of $H^{\infty}(\mathbb{B}, B(E))$. For this class of analytic functions, interpolation results of Nevanlinna-Pick or Carathéodory-Fejér type have been obtained, and closely related versions of the above functional form of the commutant lifting theorem can be proved (see [6, 13, 16, 19, 27]). Based on Agler's characterization [1] of Schur class functions on the open unit polydisc $\mathbb{D}^{n}$ in $\mathbb{C}^{n}$, corresponding interpolation and commutant lifting results have been proved over the unit polydisc in [2] and [12]. Non-commutative commutant lifting theorems for row conctractions have been proved by Popescu in $[25,26]$. As an application commutative versions of the Sarason dilation theorem have been obtained by the same author over the unit ball (see e.g. [28]).

In the present paper we replace the ball and unit polydisc by a bounded analytic polyhedron of the form

$$
D=\{z \in W ;\|d(z)\|<1\} \subset \mathbb{C}^{n},
$$

where $d: W \rightarrow \mathbb{C}^{p, q}$ is a matrix-valued analytic function on an open neighbourhood $W$ of $\bar{D}$ in $\mathbb{C}^{n}$. Instead of contractions we use the class $\mathcal{C}$ of all commuting $n$-tuples $T \in B(H)^{n}$ on a fixed separable, infinite-dimensional Hilbert space $H$ such that $\sigma(T) \subset W$ and $\|d(T)\|<1$. Since in this situation the Taylor spectrum $\sigma(T)$ of $T$ is contained in $D$, the formula

$$
\|f\|_{\mathcal{S}}=\sup \{\|f(T)\| ; T \in \mathcal{C}\}
$$

can be used to define a contractively embedded Banach subalgebra $\mathcal{S}(d, B(E))$ of $H^{\infty}(D, B(E))$, called the Schur space on $D$. We replace the Hardy space by Hilbert spaces $\mathcal{H}$ of analytic functions on $D$ defined by a suitable reproducing kernel function $C$. Our main result (Theorem 3.7) shows in particular that, for each $M_{z}^{*}$-invariant finite- 
dimensional subspace $M \subset \mathcal{H} \otimes E$, the operators $X$ in the commutant of the compression $T=P_{M} M_{z} \mid M \in B(M)^{n}$, which possess a lifting to a multiplication operator $M_{f}$ given by a Schur class function $f \in \mathcal{S}(d, B(E))$ with Schur norm $\|f\|_{\mathcal{S}} \leq 1$, can be characterized by a positivity condition which is formulated in terms of the reproducing kernel $C$ and the boundary function $d$ of the domain $D$.

The results obtained in this way apply in particular to the ball, the polydisc and, furthermore, to all classical symmetric domains of type I, II and III. Indeed, in these cases suitable examples of reproducing kernel Hilbert spaces satisfying all our conditions are known to exist. As an application we derive interpolation results for functions $f$ in $\mathcal{S}(d, B(E))$ of Nevanlinna-Pick and Carathéodory-Fejér type on these domains.

The paper continues joint work [4,5] of the first named author with D. Timotin on von Neumann type inequalities and intertwining lifting results over suitable domains in $\mathbb{C}^{n}$. We make essential use of a recent result of Ball and Bolotnikov [11] which gives various characterizations of Schur class functions on polynomial polyhedra.

0. Preliminaries. Let $H$ and $K$ be complex Hilbert spaces. We write $B(H, K)$ for the set of all bounded linear operators from $H$ to $K$ and $H \otimes K$ for the Hilbertian tensor product of $H$ and $K$. For an open set $U$ in $\mathbb{C}^{n}$ and a given Banach space $X$, we denote by $\mathcal{O}(U, X)$ the Fréchet space of all analytic $X$-valued functions on $U$. If $T \in B(H)^{n}$ is a commuting tuple of bounded linear operators on $H$, then $\sigma(T)$ is defined as the Taylor spectrum of $T$, and we write $\Phi: \mathcal{O}(\sigma(T)) \rightarrow B(H), f \mapsto f(T)$, for Taylor's analytic functional calculus of $T$. For the definition and basic properties of these notions from multivariable operator theory, we refer the reader to [18] or [32].

For given Hilbert spaces $E$ and $E_{*}$ and each open neighbourhood $U$ of $\sigma(T)$, there is a unique continuous linear map

$$
\Phi_{E, E_{*}}: \mathcal{O}\left(U, B\left(E, E_{*}\right)\right) \cong \mathcal{O}(U) \tilde{\otimes} B\left(E, E_{*}\right) \rightarrow B\left(H \otimes E, H \otimes E_{*}\right)
$$

with the property that $\Phi_{E, E_{*}}(f \otimes A)=f(T) \otimes A$ for $f \in \mathcal{O}(U)$ and $A \in B\left(E, E_{*}\right)$. For simplicity, we write again $f(T)$ instead of $\Phi_{E, E_{*}}(f)$. If $S \subset \mathbb{C}^{n}$ is an arbitrary subset, then we define $\tilde{S}=\left\{z \in \mathbb{C}^{n} ; \bar{z} \in S\right\}$. For $f \in \mathcal{O}\left(U, B\left(E, E_{*}\right)\right)$, the function

$$
\tilde{f}: \tilde{U} \rightarrow B\left(E_{*}, E\right), \quad \tilde{f}(z)=f(\bar{z})^{*}
$$

is analytic again. An elementary exercise, using the corresponding scalar-valued result and the density of the linear span of all elementary tensors, shows that the identity $\tilde{f}\left(T^{*}\right)=f(T)^{*}$ holds for all functions $f \in \mathcal{O}\left(U, B\left(E, E_{*}\right)\right)$.

If $p, q$ are positive integers, then we identify the space $B\left(H^{q}, H^{p}\right)$ of all bounded linear operators from $H^{q}$ to $H^{p}$ with the space $B(H)^{p, q}$ of all $(p \times q)$-operator matrices with entries in $B(H)$. For $p=q$, we regard the map

$$
\operatorname{tr}: B(H)^{p, p} \rightarrow B(H), \quad\left(A_{i j}\right) \mapsto \sum_{j=1}^{p} A_{j j}
$$

as a generalization of the ordinary trace for scalar matrices.

Let $\Lambda$ be an arbitrary set. An operator-valued function $K: \Lambda \times \Lambda \rightarrow B(H)$ is called positive definite if $\sum_{i, j=1}^{s}\left\langle K\left(\lambda_{i}, \lambda_{j}\right) c_{i}, c_{j}\right\rangle \geq 0$ whenever $s$ is a positive integer, $\lambda_{1}, \ldots, \lambda_{s} \in \Lambda$ 
and $c_{1}, \ldots, c_{s} \in H$. By a well-known theorem of Kolmogorov and Aronszajn a function $K: \Lambda \times \Lambda \rightarrow B(H)$ is positive definite if and only if there is a Hilbert space $G$ and a function $k: \Lambda \rightarrow B(H, G)$ such that $K(\lambda, \mu)=k(\mu)^{*} k(\lambda)$ for $\lambda, \mu \in \Lambda$.

1. Functional Hilbert spaces. Let $D \subset \mathbb{C}^{n}$ be an open set with $0 \in D$. Throughout this note we shall denote by $\mathcal{H}$ a fixed functional Hilbert space consisting of analytic functions on $D$. More precisely, $\mathcal{H}$ is a Hilbert space consisting of complex-valued analytic functions on $D$ such that all point evaluations

$$
\delta_{w}: \mathcal{H} \rightarrow \mathbb{C}, \quad f \mapsto f(w) \quad(w \in D)
$$

are continuous. For each point $w \in D$, there is a unique function $C_{w} \in \mathcal{H}$ such that

$$
\left\langle f, C_{w}\right\rangle=f(w) \quad(f \in \mathcal{H}) .
$$

The map $D \rightarrow \mathcal{H}, w \mapsto C_{w}$, is weakly anti-analytic, and hence it is anti-analytic as a map with values in $\mathcal{H}$. Consequently, on the open set $\Delta=\{(z, \bar{w}) ; z, w \in D\} \subset \mathbb{C}^{2 n}$, we can define an analytic function $C: \Delta \rightarrow \mathbb{C}$ by

$$
C(z, w)=C_{\bar{w}}(z) .
$$

In addition, we shall suppose that the domain $D$ and the functional Hilbert space $\mathcal{H}$ satisfy the following conditions:

(i) $\mathcal{H}$ contains the constant functions and $\|1\|=1$,

(ii) the coordinate functions are multipliers of $\mathcal{H}$, and the tuple $Z=\left(Z_{1}, \ldots, Z_{n}\right) \in$ $B(\mathcal{H})^{n}$ consisting of the multiplication operators $Z_{j}: \mathcal{H} \rightarrow \mathcal{H}, f \mapsto z_{j} f$, has the property that $\sigma(Z)=\bar{D}$,

(iii) $\bar{D}$ is polynomially convex and the polynomials form a dense subset of $\mathcal{H}$,

(iv) the function $C$ has no zeros, $C_{0} \equiv 1$ and $C^{-1}$ extends to a holomorphic function defined on an open neighbourhood of $\bar{\Delta}$.

Typical examples of analytic functional Hilbert spaces satisfying all these conditions are the Bergman (or Hardy) spaces on the classical symmetric domains (see Chapter IV in $[22])$.

For a given Hilbert space $E$, we shall identify the Hilbertian tensor product $\mathcal{H} \otimes E$ with a linear subspace of the space $\mathcal{O}(D, E)$ of all $E$-valued analytic functions on $D$ via the injective linear map

$$
j: \mathcal{H} \otimes E \rightarrow \mathcal{O}(D, E), \quad(j h)(z)=\left(\delta_{z} \otimes 1_{E}\right)(h) .
$$

Let $E, E$. be fixed Hilbert spaces, and let $f \in \mathcal{O}(U, B(E, E)$.$) be an analytic function$ defined on an open set $U \supset \bar{D}$. Then the multiplication operator

$$
T_{f}: \mathcal{H} \otimes E \rightarrow \mathcal{H} \otimes E . \quad h \mapsto f h
$$

is well defined and continuous linear. To verify this, it suffices to show that

$$
(f(Z) h)(z)=f(z) h(z) \quad(h \in \mathcal{H} \otimes E, z \in D),
$$

where $f(Z)$ is formed with the operator-valued analytic functional calculus explained in the preliminaries. Since both sides of the above equation depend in a continuous bilinear 
way on $f$ and $h$, it suffices to consider the case where $f \in \mathcal{O}(U)$ and $h \in \mathcal{H}$. To settle this case, one can use the observation that $Z_{j}^{*} C_{z}=\bar{z}_{j} C_{z}(1 \leq j \leq n)$ to deduce that

$$
(f(Z) h)(z)=\left\langle h, f(Z)^{*} C_{z}\right\rangle=\left\langle h, \tilde{f}\left(Z^{*}\right) C_{z}\right\rangle=\left\langle h, \tilde{f}(\bar{z}) C_{z}\right\rangle=f(z) h(z) .
$$

For given Hilbert spaces $E$ and $E_{*}$, we consider the multiplier space

$$
M\left(E, E_{*}\right)=\left\{f \in \mathcal{O}\left(D, B\left(E, E_{*}\right)\right) ; f \mathcal{H} \otimes E \subset \mathcal{H} \otimes E_{*}\right\} .
$$

By the closed graph theorem each function $f \in M\left(E, E_{*}\right)$ induces a continuous linear multiplication operator $T_{f}: \mathcal{H} \otimes E \rightarrow \mathcal{H} \otimes E_{*}, g \mapsto f g$. The space $M\left(E, E_{*}\right)$ becomes a Banach space relative to the multiplier norm $\|f\|=\left\|T_{f}\right\|$.

A closed subspace $M \subset \mathcal{H} \otimes E$ will be called $*$-invariant if $\left(Z_{j} \otimes 1_{E}\right)^{*} M \subset M$ for $j=1, \ldots, n$.

LEMma 1.1. Suppose that $M \subset \mathcal{H} \otimes E$ is a *-invariant closed subspace.

(a) The compression $T=P_{M}\left(Z \otimes 1_{E}\right) \mid M \in B(M)^{n}$ is a commuting tuple on $M$ such that $\sigma(T) \subset \bar{D}$ such that

$$
f\left(Z \otimes 1_{E}\right)^{*} M \subset M \quad \text { and } \quad f(T)=P_{M} f\left(Z \otimes 1_{E}\right) \mid M
$$

holds for each function $f \in \mathcal{O}(\bar{D})$.

(b) For $h \in \mathcal{H} \otimes E, z \in D$ and $x \in E$, we have the identity

$$
\left\langle h, C_{z} \otimes x\right\rangle=\langle h(z), x\rangle .
$$

(c) For $f \in M\left(E, E_{*}\right), z \in D$ and $x \in E_{*}$ it follows that

$$
T_{f}^{*}\left(C_{z} \otimes x\right)=C_{z} \otimes f(z)^{*} x \text {. }
$$

Proof. (a) Since the adjoint $T^{*}=Z^{*} \otimes 1_{E} \mid M$ is a commuting tuple, the same is true for $T$. The approximate point spectrum of $T^{*}$ satisfies

$$
\sigma_{\pi}\left(T^{*}\right) \subset \sigma_{\pi}\left(Z^{*} \otimes 1_{E}\right) \subset \sigma\left(Z^{*} \otimes 1_{E}\right)=\sigma\left(Z^{*}\right)=\{\bar{z} ; z \in \bar{D}\} .
$$

Since the Taylor spectrum is always contained in the polynomially convex hull of the approximate point spectrum $([30])$, we conclude that $\sigma(T) \subset \bar{D}$. Let $f \in \mathcal{O}(\bar{D})$. Since $\sigma\left(Z^{*} \otimes 1_{E}\right) \cup \sigma\left(T^{*}\right) \subset\{\bar{z} ; z \in \bar{D}\}$, the space $M$ is invariant for $\tilde{f}\left(Z^{*} \otimes 1_{E}\right)=f\left(Z \otimes 1_{E}\right)^{*}$. Hence it follows that (cf. Lemma 2.5.8 in [18])

$$
f(T)^{*}=\tilde{f}\left(T^{*}\right)=\tilde{f}\left(Z^{*} \otimes 1_{E}\right)\left|M=f\left(Z \otimes 1_{E}\right)^{*}\right| M .
$$

(b) The observation that both sides of the claimed identity are continuous linear in $h$ reduces the assertion to the case of elementary tensors $h=h_{0} \otimes y, h_{0} \in \mathcal{H}$ and $y \in E$, where it is obvious.

(c) Apply part (b) to see that

$$
\left\langle g, T_{f}^{*}\left(C_{z} \otimes x\right)\right\rangle=\langle f(z) g(z), x\rangle=\left\langle g, C_{z} \otimes f(z)^{*} x\right\rangle
$$

for $g \in \mathcal{H} \otimes E, z \in D$ and $x \in E_{*}$.

For a commuting tuple $T \in B(H)^{n}$ on a Hilbert space $H$, we denote by

$$
M_{T}=\left(L_{T}, R_{T^{*}}\right) \in B(B(H))^{2 n}
$$

the commuting $(2 n)$-tuple consisting of the tuple $L_{T}=\left(L_{T_{1}}, \ldots, L_{T_{n}}\right)$ of left multiplication operators $L_{T_{j}}: B(H) \rightarrow B(H), X \mapsto T_{j} X$, and of the tuple $R_{T^{*}}=\left(R_{T_{1}^{*}}, \ldots, R_{T_{n}^{*}}\right)$ 
of right multiplication operators $R_{T_{j}^{*}}: B(H) \rightarrow B(H), X \mapsto X T_{j}^{*}$. It is well known (see [17]) that $\sigma\left(M_{T}\right)=\sigma(T) \times \sigma\left(T^{*}\right)$. For a given analytic function $f \in \mathcal{O}\left(\sigma(T) \times \sigma\left(T^{*}\right)\right)$, we use the notation

$$
f\left(T, T^{*}\right)=f\left(M_{T}\right)\left(1_{H}\right) \in B(H) .
$$

Suppose that $T \in B(H)^{n}$ and $S \in B(K)^{n}$ are commuting tuples of bounded operators on Hilbert spaces $H$ and $K$ and that $X \in B(H, K)$ intertwines $T$ and $S$ componentwise in the sense that

$$
X T_{i}=S_{i} X \quad(1 \leq i \leq n)
$$

Then the operator $\Delta_{X}: B(H) \rightarrow B(K), A \mapsto X A X^{*}$, intertwines the multiplication tuples $M_{T} \in B(B(H))^{2 n}$ and $M_{S} \in B(B(K))^{2 n}$ componentwise. If $f$ is an analytic complex-valued function on an open neighbourhood of $\left(\sigma(T) \times \sigma\left(T^{*}\right)\right) \cup\left(\sigma(S) \times \sigma\left(S^{*}\right)\right)$, then

$$
\Delta_{X} f\left(M_{T}\right)=f\left(M_{S}\right) \Delta_{X} \quad(\text { Lemma 2.5.8 in [18]). }
$$

LEMMA 1.2. With the notation from above, we obtain the identity

$$
\frac{1}{C}\left(Z \otimes 1_{E},\left(Z \otimes 1_{E}\right)^{*}\right)=P_{E},
$$

where $P_{E} \in B(\mathcal{H} \otimes E)$ denotes the orthogonal projection onto the subspace of all constant functions.

Proof. Our hypothesis that $C_{0} \equiv 1$ implies in particular that

$$
P_{E} f=f(0) \quad(f \in \mathcal{H} \otimes E) .
$$

Let $U \supset \sigma(Z)$ and $V \supset \sigma\left(Z^{*}\right)$ be open neighbourhoods. To prove the assertion it suffices to show that, for any function $f \in \mathcal{O}(U \times V)$, the identity

$$
\left\langle f\left(Z \otimes 1_{E}, Z^{*} \otimes 1_{E}\right) C_{\lambda} \otimes x, C_{\mu} \otimes y\right\rangle=f(\mu, \bar{\lambda}) C(\mu, \bar{\lambda})\langle x, y\rangle
$$

holds for all $\lambda, \mu \in D$ and $x, y \in E$. Since both sides of this equation are continuous linear in $f$, we may suppose that $f=g \otimes h$ with $g \in \mathcal{O}(U)$ and $h \in \mathcal{O}(V)$. In this case, we obtain that

$$
\begin{aligned}
f\left(Z \otimes 1_{E}, Z^{*} \otimes 1_{E}\right) & =g\left(L_{Z \otimes 1_{E}}\right) h\left(R_{Z^{*} \otimes 1_{E}}\right)\left(1_{\mathcal{H} \otimes E}\right) \\
& =L_{g\left(Z \otimes 1_{E}\right)} R_{h\left(Z^{*} \otimes 1_{E}\right.}\left(1_{\mathcal{H} \otimes E}\right)=g\left(Z \otimes 1_{E}\right) h\left(Z^{*} \otimes 1_{E}\right) \\
& =\left(g(Z) h\left(Z^{*}\right)\right) \otimes 1_{E} .
\end{aligned}
$$

Thus the proof is completed by the observation that

$$
\left\langle h\left(Z^{*}\right) C_{\lambda}, g(Z)^{*} C_{\mu}\right\rangle=h(\bar{\lambda}) g(\mu)\left\langle C_{\lambda}, C_{\mu}\right\rangle=(g \otimes h)(\mu, \bar{\lambda}) C(\mu, \bar{\lambda}) .
$$

Let $M \subset \mathcal{H} \otimes E$ and $M_{*} \subset \mathcal{H} \otimes E_{*}$ be $*$-invariant closed subspaces. Then

$$
T=P_{M}\left(Z \otimes 1_{E}\right)\left|M \in B(M)^{n}, \quad T_{*}=P_{M_{*}}\left(Z \otimes 1_{E_{*}}\right)\right| M_{*} \in B\left(M_{*}\right)^{n}
$$

are commuting $n$-tuples with $\sigma(T) \cup \sigma\left(T_{*}\right) \subset \bar{D}$. For every function $f \in \mathcal{O}(\bar{\Delta})$ and every operator $X \in B(\mathcal{H} \otimes E)$, the identity

$$
f\left(M_{T}\right)\left(P_{M} X \mid M\right)=P_{M}\left[f\left(M_{Z \otimes 1_{E}}\right)(X)\right] \mid M
$$

holds. Indeed, it suffices to check this identity for functions $f$ of the form

$$
f=g \otimes h, \quad g \in \mathcal{O}(U), h \in \mathcal{O}(V),
$$


where $U \supset \sigma(Z)$ and $V \supset \sigma\left(Z^{*}\right)$ are fixed open neighbourhoods. In this case the assertion easily follows as an application of Lemma 1.1(a).

Proposition 1.3. For every operator $X \in B\left(M, M_{*}\right)$ which intertwines the commuting tuples $T \in B(M)^{n}$ and $T_{*} \in B\left(M_{*}\right)^{n}$ componentwise, we have the identity

$$
\left\langle\frac{1}{C}\left(M_{T_{*}}\right)\left(X X^{*}\right) h, k\right\rangle=\left\langle P_{E} X^{*} h, X^{*} k\right\rangle
$$

for all $h, k \in M_{*}$.

Proof. Using Lemma 1.2 we obtain the identity

$$
\frac{1}{C}\left(M_{T_{*}}\right)\left(X X^{*}\right)=\Delta_{X}\left(\frac{1}{C}\left(T, T^{*}\right)\right)=\Delta_{X}\left(P_{M} P_{E} \mid M\right),
$$

which is equivalent to the assertion.

2. Fractional transforms and the Schur class. Throughout this section we make the assumption that the open set $D \subset \mathbb{C}^{n}$ is a generalized analytic polyhedron in the sense that there are an open neighbourhood $W$ of $\bar{D}$ and an analytic matrix-valued function $d=\left(d_{j k}\right): W \rightarrow B\left(\mathbb{C}^{q}, \mathbb{C}^{p}\right) \cong \mathbb{C}^{p, q}$ such that $d(0)=0$ and

$$
D=\{z \in W ;\|d(z)\|<1\} .
$$

Let us fix a separable infinite-dimensional Hilbert space $H$. For a given commuting tuple $X \in B(H)^{n}$ with $\sigma(X) \subset W$, the operator-valued analytic functional calculus of $X$ applied to the function $d$ gives rise to the matrix-operator

$$
d(X)=\left(d_{j k}(X)\right) \in B\left(H^{q}, H^{p}\right) .
$$

Using the spectral mapping theorem for Taylor's analytic functional calculus, one can prove the following result.

Lemma 2.1. Let $X \in B(H)^{n}$ be a commuting tuple with $\sigma(X) \subset W$. Then

$$
\sup _{z \in \sigma(X)}\|d(z)\| \leq\|d(X)\|
$$

This result was proved in [5] for the case that the coefficients $d_{j k}$ of $d$ are polynomial functions. Since the same proof, without any changes, applies to our more general situation, we omit the details. Note that, if $\|d(X)\|<1$ in the setting of Lemma 2.1, then $\sigma(X) \subset D$. Hence, for any operator-valued analytic function $f \in \mathcal{O}\left(D, B\left(E, E_{*}\right)\right)$ with given Hilbert spaces $E$ and $E_{*}$, we can form the operator

$$
f(X) \in B\left(H \otimes E, H \otimes E_{*}\right) .
$$

Define $\mathcal{C}$ as the set of all commuting tuples $X \in B(H)^{n}$ with $\sigma(X) \subset W$ and $\|d(X)\|<1$. For $f \in \mathcal{O}\left(D, B\left(E, E_{*}\right)\right)$, we call

$$
\|f\|_{\mathcal{S}}=\sup \{\|f(X)\| ; X \in \mathcal{C}\}
$$

the Schur norm of $f$. Since, for each point $z \in D$, the $n$-tuple $z 1=\left(z_{1} 1_{H}, \ldots, z_{n} 1_{H}\right)$ belongs to $\mathcal{C}$ and since $f(z 1)=1_{H} \otimes f(z)$ for each function $f \in \mathcal{O}\left(D, B\left(E, E_{*}\right)\right)$, it follows that $\|f\|_{\mathcal{S}} \geq\|f\|_{\infty, D}$ for each such function $f$. 
The linear space

$$
\mathcal{S}\left(d, B\left(E, E_{*}\right)\right)=\left\{f \in \mathcal{O}\left(D, B\left(E, E_{*}\right)\right) ;\|f\|_{\mathcal{S}}<\infty\right\}
$$

becomes a Banach space if equipped with the Schur norm $\|\cdot\|_{\mathcal{S}}$. Its unit ball

$$
\mathcal{S}_{d}\left(E, E_{*}\right)=\left\{f \in \mathcal{O}\left(D, B\left(E, E_{*}\right)\right) ;\|f\|_{\mathcal{S}} \leq 1\right\}
$$

is called the $B\left(E, E_{*}\right)$-valued Schur class on $D$ (with respect to $d$ ). In the scalar case $E=E_{*}=\mathbb{C}$, we simply write $\mathcal{S}_{d}$ instead of $\mathcal{S}_{d}(\mathbb{C}, \mathbb{C})$.

Let $L$ and $L_{*}$ be Hilbert spaces, and let the matrix operator

$$
U=\left(\begin{array}{ll}
a & b \\
c & d
\end{array}\right) \in B\left(L \oplus E, L_{*} \oplus E_{*}\right)
$$

be a contraction. We shall use the well-known and elementary fact that, for each bounded operator $X \in B\left(L_{*}, L\right)$ with $\|X\|<1$, the operator

$$
d+c\left(1_{L}-X a\right)^{-1} X b \in B\left(E, E_{*}\right)
$$

is a contraction again. Fix an arbitrary Hilbert space $K$. With $L=K^{p} \cong \mathbb{C}^{p} \otimes K$ and $L_{*}=K^{q} \cong \mathbb{C}^{q} \otimes K$, and with $X$ replaced by $d_{K}(z)=d(z) \otimes 1_{K}(z \in D)$, we see that the analytic function $f_{U}: D \rightarrow B\left(E, E_{*}\right)$ defined by

$$
f_{U}(z)=d+c\left(1_{K^{p}}-d_{K}(z) a\right)^{-1} d_{K}(z) b
$$

is sup-norm bounded by one. To keep the notation simpler, we shall usually write $d(z)$ instead of $d_{K}(z)$ again.

For the case that $d$ is a polynomial function, the following result is contained in [5].

Proposition 2.2. Let $K$ be a Hilbert space. Suppose that the matrix operator

$$
U=\left(\begin{array}{ll}
a & b \\
c & d
\end{array}\right) \in B\left(K^{p} \oplus E, K^{q} \oplus E_{*}\right)
$$

is a contraction. Then $f_{U} \in \mathcal{S}_{d}\left(E, E_{*}\right)$.

Proof. For completeness, we indicate the elementary proof. It suffices to observe that, for any given tuple $X \in \mathcal{C}$, the operator

$$
f_{U}(X)=1_{H} \otimes d+\left(1_{H} \otimes c\right)\left(1_{H \otimes K^{p}}-d_{K}(X)\left(1_{H} \otimes a\right)\right)^{-1} d_{K}(X)\left(1_{H} \otimes b\right)
$$

is a contraction again. The reason is that the matrix-operator obtained from $U$ by replacing its coefficients by the tensor products with the identity operator on $H$ is a contraction again.

Our next aim is to derive some useful characterizations of Schur class functions, which in particular show that the converse of Proposition 2.2 holds. For the case that the coefficients of $d$ are polynomial functions, this result is contained in [11]. Since our setting is slightly more general, we indicate the main ideas.

Lemma 2.3. Let $A \in B\left(H^{r}\right)=B(H)^{r, r}$ be a positive operator. Then

$$
\|A\| \leq r\|\operatorname{tr} A\|
$$

Proof. Using the Cauchy-Schwarz inequality for the positive definite map

$$
\{1, \ldots, r\}^{2} \rightarrow B(H), \quad(i, j) \mapsto A_{j i}
$$


one obtains the estimate

$$
\left\|A_{j i}\right\| \leq\left(\left\|A_{i i}\right\|\left\|A_{j j}\right\|\right)^{1 / 2} \leq \max _{k}\left\|A_{k k}\right\|
$$

for $i, j=1, \ldots, r$. On the other hand, for any fixed index $k \in\{1, \ldots, r\}$, we have

$$
\left\|A_{k k}\right\| \leq \sup _{\|x\|=1} \max _{i}\left\langle A_{i i} x, x\right\rangle \leq\|\operatorname{tr} A\|
$$

To complete the proof it suffices to recall that $\|A\| \leq r \max _{j, i}\left\|A_{j i}\right\|$.

Let $\delta: S \rightarrow B\left(\mathbb{C}^{s}, \mathbb{C}^{r}\right)$ be a map on an arbitrary set $S$ such that $\|\delta(z)\|<1$ for $z \in S$. Lemma 2.4. Suppose that $\Gamma: S \times S \rightarrow B\left(H^{r}\right)$ is positive definite such that

$$
F: S \rightarrow B(H), \quad F(z)=\operatorname{tr}\left(\left(1-\delta(z) \delta(z)^{*}\right) \Gamma(z)\right)
$$

is sup-norm bounded by one. Then the estimate

$$
\|\Gamma(z, w)\| \leq r\left(\left(1-\|\delta(z)\|^{2}\right)\left(1-\|\delta(w)\|^{2}\right)\right)^{-(1 / 2)}
$$

holds for all $z, w \in S$.

Proof. By Lemma 2.3 we obtain that

$$
\begin{aligned}
\|\Gamma(z, z)\| & \leq\left\|\left(1-\delta(z) \delta(z)^{*}\right)^{-1 / 2}\right\|^{2}\left\|\left(1-\delta(z) \delta(z)^{*}\right)^{1 / 2} \Gamma(z, z)\left(1-\delta(z) \delta(z)^{*}\right)^{1 / 2}\right\| \\
& \leq r\left\|\left(1-\delta(z) \delta(z)^{*}\right)^{-1}\right\|\|F(z)\| \leq r\left(1-\|\delta(z)\|^{2}\right)^{-1}
\end{aligned}
$$

for all $z \in S$. To complete the proof it suffices to apply the Cauchy-Schwarz inequality to the positive definite map $\Gamma$.

After these preparations we can prove our version of the theorem from Ball and Bolotnikov [11].

ThEOREM 2.5. Let $S \subset D$ and $f: S \rightarrow B\left(E, E_{*}\right)$ be arbitrary. Then the following are equivalent:

(i) $f$ extends to a map in $\mathcal{S}_{d}\left(E, E_{*}\right)$;

(ii) there is a positive definite map $\Gamma: S \times S \rightarrow B\left(E^{q}\right)$ such that

$$
1-f(w)^{*} f(z)=\operatorname{tr}\left(\left(1-d^{t}(z) d^{t}(w)^{*}\right) \Gamma(z, w)\right) \quad(z, w \in S) ;
$$

(iii) there exist a Hilbert space $\mathcal{G}$ and a map $G: S \rightarrow B\left(E, \mathcal{G}^{q}\right)$ such that

$$
1-f(w)^{*} f(z)=G(w)^{*}\left(1-d(w)^{*} d(z)\right) G(z) \quad(z, w \in S) ;
$$

(iv) there exist a Hilbert space $K$ and a unitary operator

$$
U=\left(\begin{array}{ll}
a & b \\
c & d
\end{array}\right) \in B\left(K^{p} \oplus E, K^{q} \oplus E_{*}\right)
$$

such that $f(z)=d+c d(z)\left(1_{K^{q}}-a d(z)\right)^{-1} b$ for $z \in S$;

(ii) $^{\prime}$ there is a positive definite map $\Gamma: S \times S \rightarrow B\left(E_{*}^{p}\right)$ such that

$$
1-f(w) f(z)^{*}=\operatorname{tr}\left(\left(1-d^{t}(z)^{*} d^{t}(w)\right) \Gamma(z, w)\right) \quad(z, w \in S) ;
$$

(iii)' there exist a Hilbert space $\mathcal{G}$ and a map $G: S \rightarrow B\left(\mathcal{G}^{p}, E_{*}\right)$ such that

$$
1-f(w) f(z)^{*}=G(w)\left(1-d(w) d(z)^{*}\right) G(z)^{*} \quad(z, w \in S) ;
$$


(iv)' there exist a Hilbert space $K$ and a unitary operator $U$ as in condition (iv) such that $f=f_{U}$ on $S$.

Proof. (i) $\Rightarrow$ (ii). Let $f \in \mathcal{S}_{d}\left(E, E_{*}\right)$ be a Schur class function, and let $S \subset D$ be finite. Set $\delta(z)=d^{t}(z)$. Suppose that $\operatorname{dim}(E)<\infty$. It suffices to show that in this case there is a positive definite map $\Gamma$ as in condition (ii). Then the general case can be deduced by choosing, for each finite set $M \subset D$ and each finite-dimensional subspace $F \subset E$, a positive definite map $\Gamma_{M, F}: M \times M \rightarrow B\left(F^{q}\right)$ such that

$$
P_{F}\left(1-f(w)^{*} f(z)\right) \mid F=\operatorname{tr}\left(\left(1-\delta(z) \delta(w)^{*}\right) \Gamma_{M, F}(z, w)\right)
$$

holds for $z, w \in M$. The trivial extensions $\Delta_{M, F}: D \times D \rightarrow B\left(E^{q}\right)$ defined by setting $\Delta_{M, F}=0$ on $(D \times D) \backslash(M \times M)$ and

$$
\Delta_{M, F}(z, w)=\Gamma_{M, F}(z, w) P_{F^{q}} \quad(z, w \in M)
$$

form a net in the compact Hausdorff space

$$
\prod_{(z, w) \in D \times D}\left(\left\{X \in B\left(E^{q}\right) ;\|X\| \leq r(z, w)\right\}, \tau_{\mathrm{WOT}}\right),
$$

where $r(z, w)$ are suitable real numbers which can be chosen with the help of Lemma 2.4 and $\tau_{\text {WOT }}$ refers to the weak operator topology. It is elementary to check that the limit $\Gamma$ of any convergent subnet of $\left(\Delta_{M, F}\right)$ will give a representation of $1-f(w)^{*} f(z)$ on $D \times D$ as in condition (ii).

Thus suppose that $S \subset D$ is finite and that $\operatorname{dim}(E)<\infty$. To prove that (i) implies (ii) in this case we use a separation argument due to Agler [1]. The set $V=B(E)^{S \times \tilde{S}}$ of all functions $S \times \tilde{S} \rightarrow B(E)$ is a finite-dimensional normed space with respect to the sup-norm. It suffices to show that the function

$$
F: S \times \tilde{S} \rightarrow B(E), \quad F(z, w)=1-f(\bar{w})^{*} f(z)
$$

belongs to the subset $C \subset V$ consisting of all functions $g \in V$ for which there is a positive definite map $\Gamma: S \times S \rightarrow B\left(E^{q}\right)$ such that

$$
g(z, \bar{w})=\operatorname{tr}\left(\left(1-\delta(z) \delta(w)^{*}\right) \Gamma(z, w)\right) \quad(z, w \in S) .
$$

By Lemma 2.4 the set $C$ is a closed proper cone in $V$. Assume that $F \notin C$. Since $F$ and the elements of $C$ are self-adjoint with respect to the involution on $V$ defined by

$$
g^{*}(z, w)=g(\bar{w}, \bar{z})^{*},
$$

a well-known separation theorem (Theorem 2.7 in [23]) allows us to choose a linear functional $L: V \rightarrow \mathbb{C}$ such that $L>0$ on $C \backslash\{0\}$ and $L(F)<0$. Let $H_{0}=B(E, \mathbb{C})^{S}$ be the vector space of all functions $g: S \rightarrow B(E, \mathbb{C})$. For $g, h \in H_{0}$, define a function $g \times h$ in $V$ by

$$
g \times h(z, w)=h(\bar{w})^{*} g(z) .
$$

All functions of the form $g \times g, g \in H_{0}$, belong to $C$. Indeed, by Schur's theorem the map $\Gamma: S \times S \rightarrow B(E)$ defined by

$$
\Gamma(z, w)=\sum_{m=0}^{\infty}\left(\left\langle\left(d_{k 1}(z)\right)_{k},\left(d_{k 1}(w)\right)_{k}\right\rangle_{\mathbb{C}^{p}}\right)^{m} g(w)^{*} g(z)
$$


is positive definite and satisfies

$$
g \times g(z, \bar{w})=\operatorname{tr}\left(\left(1-\delta(z) \delta(w)^{*}\right) \pi_{1}^{*} \Gamma(z, w) \pi_{1}\right) \quad(z, w \in S),
$$

where $\pi_{1}: E^{q} \rightarrow E$ is the projection onto the first coordinate. Thus $H_{0}$ is a finitedimensional Hilbert space relative to the inner product

$$
\langle g, h\rangle=L(g \times h) .
$$

The tuple $X \in L\left(H_{0}\right)^{n}$ defined by $\left(X_{j} g\right)(z)=z_{j} g(z)$ for $z \in S$ and $j=1, \ldots, n$ is commutative with $\sigma(X)=S$. Since for each non-zero element $h \in H_{0}^{q}$ the relation

$$
\begin{aligned}
\|h\|^{2}-\|d(X) h\|^{2} & =L\left(\sum_{i, j=1}^{q}\left(\delta_{i j}-\sum_{k=1}^{p} d_{k j}(z)\left(d_{k i}\right) \sim(w)\right) h_{i}(\bar{w})^{*} h_{j}(z)\right) \\
& =L\left(\operatorname{tr}\left[\left(1-\delta(z) \delta(\bar{w})^{*}\right) \Gamma_{h}(z, \bar{w})\right]\right)>0
\end{aligned}
$$

holds with $\Gamma_{h}(z, w)=\left(h_{i}(w)^{*} h_{j}(z)\right)_{1 \leq i, j \leq q}$, we conclude that $\|d(X)\|<1$.

Since by hypothesis $f \in \mathcal{S}_{d}\left(E, E_{*}\right)$, it follows that $\|f(X)\| \leq 1$. For fixed vectors $g, h \in H_{0}$ and $x, y \in E$, the identity

$$
\langle u(X) g \otimes x, v(X) h \otimes y\rangle_{H_{0} \otimes E_{*}}=L\left(\langle u(z) x, v(\bar{w}) y\rangle_{E_{*}} g \times h(z, w)\right)
$$

holds for each pair of functions $u, v \in \mathcal{O}\left(D, B\left(E, E_{*}\right)\right)$. It suffices to check this assertion for elementary tensors $u=u_{0} \otimes A, v=v_{0} \otimes B$, which is straightforward.

Fix an orthonormal basis $\left(e_{j}\right)_{j=1}^{r}$ of $E$ and define, for $j=1, \ldots, r$,

$$
f_{j}: S \rightarrow B(E, \mathbb{C}), \quad f_{j}(z) x=\left\langle x, e_{j}\right\rangle .
$$

Then $f_{i} \times f_{j} \equiv\left\langle\cdot, e_{i}\right\rangle e_{j}$ on $S \times \tilde{S}$. The identity

$$
F(z, w)=\sum_{i, j=1}^{r}\left\langle F(z, w) e_{i}, e_{j}\right\rangle\left\langle\cdot, e_{i}\right\rangle e_{j},
$$

valid for all $(z, w) \in S \times \tilde{S}$, implies that

$$
\begin{aligned}
L(F) & =L\left(\sum_{j=1}^{r}\left(f_{j} \times f_{j}\right)(z, w)-\sum_{i, j=1}^{r}\left\langle f(z) e_{i}, f(\bar{w}) e_{j}\right\rangle f_{i} \times f_{j}(z, w)\right) \\
& =\left\|\sum_{j=1}^{r} f_{j} \otimes e_{j}\right\|_{H_{0} \otimes E}^{2}-\left\|f(X)\left(\sum_{j=1}^{r} f_{j} \otimes e_{j}\right)\right\|_{H_{0} \otimes E_{*}}^{2} \geq 0 .
\end{aligned}
$$

This contradiction completes the proof of the implication (i) $\Rightarrow$ (ii).

(ii) $\Rightarrow$ (iii). Suppose that $\Gamma$ is given as in condition (ii). Then there is a Hilbert space $\mathcal{G}$ and a map $\tilde{G}: S \rightarrow B\left(E^{q}, \mathcal{G}\right)$ such that $\Gamma(z, w)=\tilde{G}(w)^{*} \tilde{G}(z)$ for $z, w \in S$. Write $\tilde{G}(z)=\left(G_{1}(z), \ldots, G_{q}(z)\right)$ and define

$$
G: S \rightarrow B\left(E, \mathcal{G}^{q}\right), \quad G(z)=\left(G_{1}(z), \ldots, G_{q}(z)\right)^{t} .
$$

Then, with $\delta(z)=d^{t}(z)$, we obtain that

$$
\begin{aligned}
\operatorname{tr}\left(\left(1-\delta(z) \delta(w)^{*}\right) \Gamma(z, w)\right) & =\sum_{j=1}^{q} G_{j}(w)^{*} G_{j}(z)-\sum_{i, j=1}^{q} G_{i}(w)^{*}\left(\sum_{k=1}^{p} \overline{d_{k i}(w)} d_{k j}(z)\right) G_{j}(z) \\
& =G(w)^{*}\left(1-d(w)^{*} d(z)\right) G(z)
\end{aligned}
$$

for $z, w \in S$. 
(iii) $\Rightarrow$ (iv). $\quad$ Let $G$ be a map as in condition (iii). Then, for $z, w \in S$ and $x, y \in E$, we obtain the identity

$$
\langle d(z) G(z) x, d(w) G(w) y\rangle_{\mathcal{G}^{p}}+\langle x, y\rangle_{E}=\langle G(z) x, G(w) y\rangle_{\mathcal{G}^{q}}+\langle f(z) x, f(w) y\rangle_{E_{*}} .
$$

Hence there exist a Hilbert space $K \supset \mathcal{G}$ and a unitary operator $U$ of the form described in condition (iv) such that

$$
U\left(\begin{array}{c}
d(z) G(z) x \\
x
\end{array}\right)=\left(\begin{array}{c}
G(z) x \\
f(z) x
\end{array}\right)
$$

for all $z \in S$ and $x \in E$. By solving the corresponding system of linear equations

$$
a d(z) G(z)+b=G(z), \quad c d(z) G(z)+d=f(z),
$$

we obtain that, for all $z \in S$,

$$
f(z)=d+c d(z)\left(1_{K^{q}}-a d(z)\right)^{-1} b .
$$

(iv) $\Rightarrow(\text { iv) })^{\prime} \quad$ Suppose that $U$ represents $f$ as in condition (iv). Using the identity

$$
\left(1_{K^{q}}-a d(z)\right)^{-1}=1_{K^{q}}+a\left(1_{K^{p}}-d(z) a\right)^{-1} d(z)
$$

we see that

$$
\begin{aligned}
f(z) & =d+c d(z) b+c d(z) a\left(1_{K^{p}}-d(z) a\right)^{-1} d(z) b \\
& =d+c\left(1_{K^{p}}-d(z) a\right)^{-1} d(z) b .
\end{aligned}
$$

In Proposition 2.2 we proved that (iv)' implies (i). Completely analogous to the above arguments one can prove the implications (i) $\Rightarrow(\text { ii })^{\prime} \Rightarrow(\text { iii })^{\prime} \Rightarrow(\text { iv })^{\prime}$. Thus the proof of Theorem 2.5 is complete.

3. A commutant lifting theorem. Let $\mathcal{H}$ be a functional Hilbert space consisting of analytic functions defined on an open set $D$ in $\mathbb{C}^{n}$ such that $\mathcal{H}$ and $D$ satisfy the conditions described at the beginning of $\S 1$. As in $\S 2$ we suppose that there is an open neighbourhood $W$ of $\bar{D}$ and an analytic function $d: W \rightarrow B\left(\mathbb{C}^{q}, \mathbb{C}^{p}\right)$ such that $d(0)=0$ and

$$
D=\{z \in W ;\|d(z)\|<1\} .
$$

As typical examples one can choose the Bergman and Hardy spaces on the unit ball, the unit polydisc or the classical symmetric domains of types I, II and III (see [22]).

For $0<r<1$, the set $W_{r}=\{z \in W ;\|d(z)\|<1 / r\}$ is an open neighbourhood of $\bar{D}$. Recall that the operator-valued analytic functional calculus of the multiplication tuple $Z=\left(Z_{1}, \ldots, Z_{n}\right) \in B(\mathcal{H})^{n}$ gives rise to the matrix operator $d(Z) \in B\left(\mathcal{H}^{q}, \mathcal{H}^{p}\right)$. Throughout this section we make the additional assumption that

$$
\|d(Z)\| \leq 1
$$

Let $E$ and $E_{*}$ be arbitrary Hilbert spaces. It is well known ([14], [15]) that a function $f \in \mathcal{O}\left(D, B\left(E, E_{*}\right)\right)$ belongs to the closed unit ball of $M\left(E, E_{*}\right)$ with respect to the multiplier norm $\|f\|=\left\|T_{f}\right\|$ if and only if the induced map

$$
K_{f}: D \times D \rightarrow B\left(E_{*}\right), \quad(z, w) \mapsto C(w, \bar{z})\left(1_{E_{*}}-f(w) f(z)^{*}\right)
$$

is positive definite. 
LEMma 3.1. Let $E$ and $E_{*}$ be Hilbert spaces. Then $\mathcal{S}\left(d, B\left(E, E_{*}\right)\right) \subset M\left(E, E_{*}\right)$ and

$$
\left\|T_{f}\right\| \leq\|f\|_{\mathcal{S}}
$$

for all $f \in \mathcal{S}\left(d, B\left(E, E_{*}\right)\right)$.

Proof. Let $f \in \mathcal{S}_{d}\left(E, E_{*}\right)$ be a Schur class function. By Theorem 2.5 there are a Hilbert space $\mathcal{G}$ and a map $G: D \rightarrow B\left(\mathcal{G}^{p}, E_{*}\right)$ such that

$$
1-f(w) f(z)^{*}=G(w)\left(1-d(w) d(z)^{*}\right) G(z)^{*} \quad(z, w \in D) .
$$

Multiplying both sides of this equation with $C(w, \bar{z})$ and using the above multiplier characterization twice, first for $d$ and then for $f$, yields the assertion.

A second important consequence of the hypothesis that $\|d(Z)\| \leq 1$ is the following result, which should be compared with Lemma 3.2 in [12].

Lemma 3.2. Let $K$ and $E_{*}$ be Hilbert spaces. Suppose that $a \in B\left(K^{p}, K^{q}\right)$ and $c \in$ $B\left(K^{p}, E_{*}\right)$ are bounded operators with $a^{*} a+c^{*} c \leq 1_{K^{p}}$. Then, for $x \in K^{p}$, the function $\Omega x: D \rightarrow E_{*}$ defined by

$$
(\Omega x)(z)=c\left(1_{K^{p}}-d_{K}(z) a\right)^{-1} x
$$

belongs to $\mathcal{H} \otimes E_{*}$. The map $\Omega: K^{p} \rightarrow \mathcal{H} \otimes E_{*}, x \mapsto \Omega x$, is a linear contraction such that

$$
\Omega^{*}\left(C_{z} \otimes y\right)=\left(1_{K^{p}}-a^{*} d_{K}(z)^{*}\right)^{-1} c^{*} y \quad\left(z \in D, y \in E_{*}\right) .
$$

Proof. Define $\alpha=1_{\mathcal{H}} \otimes a \in B\left(\mathcal{H} \otimes K^{p}, \mathcal{H} \otimes K^{q}\right)$. Let us fix an arbitrary real number $r$ with $0<r<1$. Then the operator

$$
\delta=\delta_{r}=r d(Z) \otimes 1_{K} \in B\left(\mathcal{H}^{q} \otimes K, \mathcal{H}^{p} \otimes K\right) \cong B\left(\mathcal{H} \otimes K^{q}, \mathcal{H} \otimes K^{p}\right)
$$

satisfies $\left\|\delta_{r}\right\| \leq r$. By the remarks preceding Lemma 1.1 the functions

$$
\begin{aligned}
& \omega=\omega_{r}: W_{r} \rightarrow B\left(K^{p}, E_{*}\right), \quad z \mapsto c\left(1-r d_{K}(z) a\right)^{-1}, \\
& \varphi=\varphi_{r}: W_{r} \rightarrow B\left(K^{p}\right), \quad z \mapsto\left(1+r d_{K}(z) a\right)\left(1-r d_{K}(z) a\right)^{-1}
\end{aligned}
$$

induce multipliers $T_{\omega} \in M\left(K^{p}, E_{*}\right)$ and $T_{\varphi} \in M\left(K^{p}\right)$. Our hypotheses on $a$ and $c$ imply that

$$
\begin{aligned}
\frac{T_{\varphi}+T_{\varphi}^{*}}{2} & =\frac{1}{2}\left[(1+\delta \alpha)(1-\delta \alpha)^{-1}+\left(1-\alpha^{*} \delta^{*}\right)^{-1}\left(1+\alpha^{*} \delta^{*}\right)\right] \\
& =\left(1-\alpha^{*} \delta^{*}\right)^{-1}\left(1-\alpha^{*} \delta^{*} \delta \alpha\right)(1-\delta \alpha)^{-1} \geq\left(1-\alpha^{*} \delta^{*}\right)^{-1}\left(1-r^{2} \alpha^{*} \alpha\right)(1-\delta \alpha)^{-1} \\
& \geq r^{2}\left(1-\alpha^{*} \delta^{*}\right)^{-1}\left(1_{\mathcal{H}} \otimes c^{*}\right)\left(1_{\mathcal{H}} \otimes c\right)(1-\delta \alpha)^{-1}=r^{2} T_{\omega}^{*} T_{\omega} .
\end{aligned}
$$

In particular, it follows that

$$
r^{2}\left\|T_{\omega_{r}}\left(C_{0} \otimes x\right)\right\|^{2} \leq(1 / 2)\left\langle\left(T_{\varphi_{r}}+T_{\varphi_{r}}^{*}\right) C_{0} \otimes x, C_{0} \otimes x\right\rangle=\|x\|^{2}
$$

for $0<r<1$ and $x \in K^{p}$. Since, for $x \in K^{p}, y \in E_{*}$ and $z \in D$, we have

$$
\left\langle T_{\omega_{r}}\left(C_{0} \otimes x\right), C_{z} \otimes y\right\rangle=\left\langle x, \omega_{r}(z)^{*} y\right\rangle=\left\langle\omega_{r}(z) x, y\right\rangle,
$$

it follows that the $\operatorname{limit}_{\lim _{r} \uparrow 1}\left\langle T_{\omega_{r}}\left(C_{0} \otimes x\right), h\right\rangle$ exists for $x \in K^{p}$ and $h$ in the linear span of all vectors $C_{z} \otimes y, z \in D$ and $y \in E_{*}$. Because of

$$
\varlimsup_{r \uparrow 1}\left\|T_{\omega_{r}}\left(C_{0} \otimes x\right)\right\| \leq\|x\|
$$


the above limit exists for all $x \in K^{p}$ and $h \in \mathcal{H} \otimes E_{*}$ and can be represented by a linear contraction $\Omega^{\prime}: K^{p} \rightarrow \mathcal{H} \otimes E_{*}$ in the sense that

$$
\left\langle\Omega^{\prime} x, h\right\rangle=\lim _{r \uparrow 1}\left\langle T_{\omega_{r}}\left(C_{0} \otimes x\right), h\right\rangle \quad\left(x \in K^{p}, h \in \mathcal{H} \otimes E_{*}\right) .
$$

In view of the identity

$$
\left\langle\left(\Omega^{\prime} x\right)(z), y\right\rangle=\lim _{r \uparrow 1}\left\langle\omega_{r}(z) x, y\right\rangle=\langle(\Omega x)(z), y\rangle
$$

it is clear that $\Omega=\Omega^{\prime} \in B\left(K^{p}, \mathcal{H} \otimes E_{*}\right)$ is a well-defined contraction. The proof is completed by the observation that

$$
\left\langle\Omega^{*}\left(C_{z} \otimes y\right), x\right\rangle=\langle y,(\Omega x)(z)\rangle=\left\langle\left(1-a^{*} d_{K}(z)^{*}\right)^{-1} c^{*} y, x\right\rangle
$$

holds for all elements $y \in E_{*}, x \in K^{p}$ and $z \in D$.

Let $E$ and $E_{*}$ be complex Hilbert spaces. Suppose that $M \subset \mathcal{H} \otimes E$ and $M_{*} \subset \mathcal{H} \otimes E_{*}$ are $*$-invariant closed subspaces. Denote by

$$
T=P_{M}\left(Z \otimes 1_{E}\right)\left|M \in B(M)^{n}, \quad T_{*}=P_{M_{*}}\left(Z \otimes 1_{E_{*}}\right)\right| M_{*} \in B\left(M_{*}\right)^{n}
$$

the compressions of $Z \otimes 1_{E}$ and $Z \otimes 1_{E_{*}}$ to $M$ and $M_{*}$, respectively. Our aim is to find positivity conditions that characterize those operators $X: M \rightarrow M_{*}$ which intertwine $T$ and $T_{*}$ and which possess a lifting to a multiplier $T_{f}: \mathcal{H} \otimes E \rightarrow \mathcal{H} \otimes E_{*}$ with a Schur class symbol $f$.

For this purpose, we fix an orthonormal basis $\left(e_{k}\right)_{k \geq 0}$ of $\mathcal{H}$ consisting of polynomials such that $\mathbb{C}[z]$ is the linear span of the polynomials $e_{k}$. To see that such a sequence $\left(e_{k}\right)_{k \geq 0}$ exists, it suffices to recall that by hypothesis the polynomials form a dense subset of $\mathcal{H}$ and to apply the Gram-Schmidt orthonormalization procedure to the family $z^{\alpha}\left(\alpha \in \mathbb{N}^{n}\right)$. Define $A_{k}=e_{k}\left(T_{*}\right) \in B\left(M_{*}\right)$ for $k \geq 0$. In addition to our previous hypotheses we make the assumption that the set

$$
M_{0}=\left\{x \in M_{*} ; \sum_{k \geq 0}\left\|A_{k}^{*} x\right\|^{2}<\infty\right\}
$$

is dense in $M_{*}$. We shall see later that this condition is automatically satisfied in some important cases. Note that $M_{0} \subset M_{*}$ is a linear subspace which is invariant for each operator in the commutant of $T_{*}^{*}$. For any given operator $B \in B\left(M_{*}\right)$, the map

$$
\tilde{B}: M_{0} \times M_{0} \rightarrow \mathbb{C}, \quad \tilde{B}(x, y)=\sum_{k \geq 0}\left\langle A_{k} B A_{k}^{*} x, y\right\rangle
$$

is a well-defined sesquilinear form. More generally, let $p \geq 1$ be a fixed natural number. Then, in exactly the same way, the $p$-fold direct sums $A_{k}^{(p)} \in B\left(M_{*}^{p}\right)$ can be used to associate with each operator $B \in B\left(M_{*}^{p}\right)$ a sesquilinear form

$$
\tilde{B}: M_{0}^{p} \times M_{0}^{p} \rightarrow \mathbb{C}, \quad \tilde{B}(x, y)=\sum_{k \geq 0}\left\langle A_{k}^{(p)} B A_{k}^{*(p)} x, y\right\rangle .
$$

For $1 \leq i \leq p$, we denote by

$$
\begin{gathered}
\iota_{i}: M_{*} \rightarrow M_{*}^{p}, \quad x \mapsto\left(\delta_{i j} x\right)_{j=1}^{p}, \\
\pi_{i}: M_{*}^{p} \rightarrow M_{*}, \quad\left(x_{j}\right)_{j=1}^{p} \mapsto x_{i}
\end{gathered}
$$

the canonical inclusions and projections. 
LEMmA 3.3. Let $B=\left(B_{i j}\right) \in B\left(M_{*}^{p}\right)$ be a given operator. Then, for $\rho, \sigma=1, \ldots, q$, $\mu, \nu=1, \ldots, p$ and all vectors $x, y \in M_{0}$, we obtain the identities

(a) $\tilde{B}\left(\iota_{\nu} x, \iota_{\mu} y\right)=\left(B_{\mu \nu}\right) \sim(x, y)$,

(b) $\tilde{B}\left(d^{t}\left(T_{*}\right)^{*}\left(\iota_{\rho} x\right), d^{t}\left(T_{*}\right)^{*}\left(\iota_{\sigma} y\right)\right)=\sum_{i, j=1}^{p}\left(B_{i j}\right)^{\sim}\left(d_{j \rho}\left(T_{*}\right)^{*} x, d_{i \sigma}\left(T_{*}\right)^{*} y\right)$.

Proof. (a) By definition we have

$$
\tilde{B}\left(\iota_{\nu} x, \iota_{\mu} y\right)=\sum_{k=0}^{\infty}\left\langle A_{k}^{(p)} B A_{k}^{*(p)}\left(\iota_{\nu} x\right),\left(\iota_{\mu} y\right)\right\rangle=\sum_{k=0}^{\infty}\left\langle A_{k}\left(\pi_{\mu} B \iota_{\nu}\right) A_{k}^{*} x, y\right\rangle=\left(B_{\mu \nu}\right)^{\sim}(x, y) .
$$

(b) Define $C=d^{t}\left(T_{*}\right)^{*} \in B\left(M_{*}\right)^{p, q}$. Then as an application of part (a) we obtain that

$$
\tilde{B}\left(C\left(\iota_{\rho} x\right), C\left(\iota_{\sigma} y\right)\right)=\sum_{i, j=1}^{p} \tilde{B}\left(\iota_{j}\left(C_{j \rho} x\right), \iota_{i}\left(C_{i \sigma} y\right)\right)=\sum_{i, j=1}^{p}\left(B_{i j}\right) \sim\left(C_{j \rho} x, C_{i \sigma} y\right) .
$$

Thus the proof is complete.

For $B=\left(B_{i j}\right) \in B\left(M_{*}^{p}\right)$, we define a sesquilinear form $\tau B: M_{0} \times M_{0} \rightarrow \mathbb{C}$ by setting

$$
\tau B(x, y)=\sum_{j=1}^{p}\left(B_{j j}\right)^{\sim}(x, y)-\sum_{i, j=1}^{p} \sum_{k=1}^{q}\left(B_{i j}\right)^{\sim}\left(d_{j k}\left(T_{*}\right)^{*} x, d_{i k}\left(T_{*}\right)^{*} y\right) .
$$

Lemma 3.4. Let $\Gamma=\langle\cdot, y\rangle=B\left(M_{*}^{p}\right)$ be a positive rank-one operator given by a vector $y \in M_{*}^{p}$. Then there is a linear map $S=S_{y}: M_{0} \rightarrow \mathcal{H}^{p}$ such that

(i) $\tau \Gamma(x, x)=\|S x\|^{2}-\left\|d(Z)^{*} S x\right\|^{2}$ for all $x \in M_{0}$,

(ii) $\langle S x, q\rangle=\left\langle x, \sum_{j=1}^{p} q_{j}\left(T_{*}\right) y_{j}\right\rangle$ for all $x \in M_{0}$ and $q=\left(q_{j}\right) \in \mathbb{C}[z]^{p}$.

Proof. For $x \in M_{0}^{p}$, the map $t_{x}: \mathbb{C}[z] \rightarrow \mathbb{C}, q \mapsto\left\langle x, q\left(T_{*}\right)^{(p)} y\right\rangle$, is antilinear such that

$$
\sum_{k=0}^{\infty}\left|t_{x}\left(e_{k}\right)\right|^{2}=\sum_{k=0}^{\infty}\left\langle A_{k}^{(p)} \Gamma A_{k}^{*(p)} x, x\right\rangle=\tilde{\Gamma}(x, x)<\infty .
$$

Hence we obtain a well-defined linear map $t: M_{0}^{p} \rightarrow \mathcal{H}$ by setting

$$
t(x)=\sum_{k=0}^{\infty} t_{x}\left(e_{k}\right) e_{k} .
$$

By definition it follows that $\left\langle t(x), e_{k}\right\rangle=t_{x}\left(e_{k}\right)$ for all $k \in \mathbb{N}$. Since $\mathbb{C}[z]$ is the linear span of the polynomials $e_{k}(k \in \mathbb{N})$ and since each function $f \in \mathcal{O}(\bar{D})$ is the uniform limit of polynomials on an open neighbourhood of $\bar{D}$, we obtain that

$$
\langle t(x), f\rangle=\left\langle x, f\left(T_{*}\right)^{(p)} y\right\rangle
$$

for $x \in M_{0}^{p}$ and $f \in \mathcal{O}(\bar{D})$. Furthermore, for $x, x^{\prime} \in M_{0}^{p}$, we have

$$
\left\langle t(x), t\left(x^{\prime}\right)\right\rangle=\tilde{\Gamma}\left(x, x^{\prime}\right) .
$$

The observation that, for $r, s \in \mathcal{O}(\bar{D})$ and $x \in M_{0}^{p}$, the identity

$$
\begin{aligned}
\left\langle t\left(r\left(T_{*}\right)^{*(p)} x\right), s\right\rangle & =\left\langle r\left(T_{*}\right)^{*(p)} x, s\left(T_{*}\right)^{(p)} y\right\rangle \\
& =\left\langle x,(r s)\left(T_{*}\right)^{(p)} y\right\rangle=\langle t(x), r s\rangle=\left\langle r(Z)^{*} t(x), s\right\rangle
\end{aligned}
$$


holds, allows us to conclude that the intertwining relation

$$
t \circ r\left(T_{*}\right)^{*(p)}=r(Z)^{*} \circ t
$$

holds on $M_{0}^{p}$. Write $t$ as a row operator $t=\left(t_{1}, \ldots, t_{p}\right) \in B\left(M_{0}^{p}, \mathcal{H}\right)$ and define

$$
S: M_{0} \rightarrow \mathcal{H}^{p}, \quad x \mapsto\left(t_{j} x\right)_{j=1}^{p} .
$$

Using Lemma 3.3 (a) we obtain that

$$
\left\langle t\left(\iota_{j} x\right), t\left(\iota_{i} x^{\prime}\right)\right\rangle=\tilde{\Gamma}\left(\iota_{j} x, \iota_{i} x^{\prime}\right)=\left(\Gamma_{i j}\right) \sim\left(x, x^{\prime}\right)
$$

for $i, j=1, \ldots, p$ and $x, x^{\prime} \in M_{0}$, and hence that

$$
\begin{aligned}
\left(\Gamma_{i j}\right)^{\sim}\left(d_{j k}\left(T_{*}\right)^{*} x, d_{i k}\left(T_{*}\right)^{*} x\right) & =\left\langle t\left(d_{j k}\left(T_{*}\right)^{*(p)} \iota_{j} x\right), t\left(d_{i k}\left(T_{*}\right)^{*(p)} \iota_{i} x\right)\right\rangle \\
& =\left\langle d_{j k}(Z)^{*}(S x)_{j}, d_{i k}(Z)^{*}(S x)_{i}\right\rangle
\end{aligned}
$$

for all $i, j=1, \ldots, p, k=1, \ldots, q$ and $x \in M_{0}$. Adding up we find that

$$
\begin{aligned}
\sum_{i, j=1}^{p} \sum_{k=1}^{q}\left(\Gamma_{i j}\right)^{\sim}\left(d_{j k}\left(T_{*}\right)^{*} x, d_{i k}\left(T_{*}\right)^{*} x\right) & =\sum_{i, j=1}^{p}\left\langle\left(d(Z) d(Z)^{*}\right)_{i, j}(S x)_{j},(S x)_{i}\right\rangle \\
& =\left\|d(Z)^{*} S x\right\|^{2}
\end{aligned}
$$

for all $x \in M_{0}$. Now, condition (i) follows from the observation that

$$
\sum_{j=1}^{p}\left(\Gamma_{j j}\right)^{\sim}(x, x)=\sum_{j=1}^{p}\left\langle(S x)_{j},(S x)_{j}\right\rangle=\|S x\|^{2}
$$

for all vectors $x \in M_{0}$. At the same time we find that

$$
\begin{aligned}
\langle S x, q\rangle & =\sum_{j=1}^{p}\left\langle t\left(\iota_{j} x\right), q_{j}\right\rangle=\sum_{j=1}^{p}\left\langle\iota_{j} x, q_{j}\left(T_{*}\right)^{(p)} y\right\rangle \\
& =\sum_{j=1}^{p}\left\langle x, q_{j}\left(T_{*}\right) y_{j}\right\rangle=\left\langle x, \sum_{j=1}^{p} q_{j}\left(T_{*}\right) y_{j}\right\rangle
\end{aligned}
$$

for $x \in M_{0}$ and $q=\left(q_{j}\right) \in \mathbb{C}[z]^{p}$.

It follows from Lemma 3.4 that $\tau \Gamma$ is a positive semi-definite sesquilinear form for each positive rank-one operator $\Gamma \in B\left(M_{*}^{p}\right)$. Our next aim is to show that $\Gamma$ has to be zero if $\tau \Gamma=0$.

Lemma 3.5. Suppose that $\Gamma=\langle\cdot, y\rangle y \in B\left(M_{*}^{p}\right)$ is a positive rank-one operator. If $\tau \Gamma=0$, then $\Gamma=0$.

Proof. Choose a linear map $S: M_{0} \rightarrow \mathcal{H}^{p}$ as in Lemma 3.4. The hypothesis that $\tau \Gamma \equiv 0$ means precisely that

$$
\langle S x, S x\rangle=\left\langle d(Z) d(Z)^{*} S x, S x\right\rangle \quad\left(x \in M_{0}\right) .
$$

Since $d(Z) d(Z)^{*} \in B\left(\mathcal{H}^{p}\right)$ is a positive contraction, it follows that $d(Z) d(Z)^{*} S=S$. Because of $d(Z)^{*}\left(C_{0} \otimes \alpha\right)=C_{0} \otimes\left(d(0)^{*} \alpha\right)=0$ for $\alpha \in \mathbb{C}^{p}$ we find that

$$
\left\langle S x, C_{0} \otimes \alpha\right\rangle=\left\langle S x-d(Z) d(Z)^{*} S x, C_{0} \otimes \alpha\right\rangle=0
$$


for $x \in M_{0}$ and $\alpha \in \mathbb{C}^{p}$. Since $C_{0} \equiv 1$, part (ii) of Lemma 3.4 allows us to conclude that

$$
0=\left\langle S x, C_{0} \otimes \alpha\right\rangle=\left\langle x, \sum_{j=1}^{p} \alpha_{j} y_{j}\right\rangle=\sum_{j=1}^{p} \bar{\alpha}_{j}\left\langle x, y_{j}\right\rangle
$$

for $x \in M_{0}$ and $\alpha=\left(\alpha_{j}\right) \in \mathbb{C}^{p}$. Using the density of $M_{0}$ we deduce that $y=0$.

The fact that each positive operator $\Delta \in B\left(M_{*}^{p}\right)$ can be represented as the limit of a strongly convergent, increasing series of positive rank-one operators allows one to improve the above results considerably.

Proposition 3.6. Let $\Delta \in B\left(M_{*}^{p}\right)$ be a positive operator. Then $\tau \Delta \geq 0$, and $\tau \Delta=0$ if and only if $\Delta=0$.

Proof. Define $\gamma=\Delta^{1 / 2}$ and fix an orthonormal basis $\left(u_{i}\right)_{i \in I}$ of $M_{*}^{p}$. Then the orthogonal projections $\pi_{J}=\sum_{j \in J}\left\langle\cdot, u_{j}\right\rangle u_{j}, J \subset I$ finite, form an increasing net of positive operators converging strongly to the identity operator. Fix a vector $x \in M_{0}^{p}$ and a real number $\varepsilon>0$. Then there is a natural number $k=k(x, \varepsilon)$ such that

$$
0 \leq \tilde{\Delta}(x, x)-\sum_{j=0}^{k}\left\langle A_{j}^{(p)} \Delta A_{j}^{*(p)} x, x\right\rangle<\varepsilon / 2 .
$$

For $k$ fixed, we can choose a finite set $J_{0} \subset I$ such that

$$
0 \leq \sum_{j=0}^{k}\left(\left\langle\gamma A_{j}^{*(p)} x, \gamma A_{j}^{*(p)} x\right\rangle-\left\langle\pi_{J} \gamma A_{j}^{*(p)} x, \gamma A_{j}^{*(p)} x\right\rangle\right)<\varepsilon / 2
$$

for all finite sets $J \subset I$ containing $J_{0}$. Hence, for the same sets $J$, we have

$$
0 \leq \tilde{\Delta}(x, x)-\sum_{j=0}^{k}\left\langle\gamma \pi_{J} \gamma A_{j}^{*(p)} x, A_{j}^{*(p)} x\right\rangle<\varepsilon .
$$

Thus we obtain that $0 \leq \tilde{\Delta}(x, x)-\left(\gamma \pi_{J} \gamma\right) \sim(x, x)<\varepsilon$ for all finite sets $J \supset J_{0}$. Consequently we have shown that $\lim _{J}\left(\gamma \pi_{J} \gamma\right)^{\sim}(x, x)=\tilde{\Delta}(x, x)$ for all $x \in M_{0}^{p}$.

Fix a vector $x \in M_{0}$. Since, for all $J \subset I$ finite, the operators $\gamma \pi_{J} \gamma=\sum_{j \in J}\left\langle\cdot, \gamma u_{j}\right\rangle \gamma u_{j}$ are finite sums of positive rank-one operators, Lemma 3.4 shows that the expressions

$$
\tau\left(\gamma \pi_{J} \gamma\right)(x, x)=\sum_{j=1}^{p}\left(\gamma \pi_{J} \gamma\right)^{\sim}\left(\iota_{j} x, \iota_{j} x\right)-\sum_{k=1}^{q}\left(\gamma \pi_{J} \gamma\right)^{\sim}\left(d^{t}\left(T_{*}\right)^{*} \iota_{k} x, d^{t}\left(T_{*}\right)^{*} \iota_{k} x\right)
$$

form an increasing net of non-negative real numbers. But then

$$
\tau(\Delta)(x, x)=\lim _{J} \tau\left(\gamma \pi_{J} \gamma\right)(x, x) \geq 0
$$

If this limit is zero for each $x \in M_{0}$, then $\tau\left(\left\langle\cdot, \gamma u_{j}\right\rangle \gamma u_{j}\right) \equiv 0 \quad(j \in I)$, and by Lemma 3.5 also $\gamma u_{j}=0$ for all $j \in I$. This observation completes the proof.

Let as before $T \in B(M)^{n}$ and $T_{*} \in B\left(M_{*}\right)^{n}$ be compressions of $Z \otimes 1_{E}$ and $Z \otimes 1_{E_{*}}$ to $*$-invariant closed subspaces $M \subset \mathcal{H} \otimes E$ and $M_{*} \subset \mathcal{H} \otimes E_{*}$, respectively. Under the hypothesis that the set

$$
M_{0}=\left\{x \in M_{*} ; \sum_{k=0}^{\infty}\left\|e_{k}\left(T_{*}\right)^{*} x\right\|^{2}<\infty\right\}
$$


is dense in $M_{*}$, we characterize those operators $X \in B\left(M, M_{*}\right)$ intertwining $T$ and $T_{*}$ that possess a lifting to a multiplier with Schur class symbol.

THEOREM 3.7. Let $X \in B\left(M, M_{*}\right)$ be a bounded operator such that $X T_{i}=T_{* i} X$ for $i=1, \ldots, n$. Then the following conditions are equivalent:

(i) there exists a function $F \in \mathcal{S}_{d}\left(E, E_{*}\right)$ such that $X P_{M}=P_{M_{*}} T_{F}$;

(ii) there exists a positive operator $\Gamma=\left(\Gamma_{i j}\right) \in B\left(M_{*}^{p}\right)$ such that

$$
\frac{1}{C}\left(M_{T_{*}}\right)\left(1-X X^{*}\right)=\sum_{j=1}^{p} \Gamma_{j j}-\sum_{i, j=1}^{p} \sum_{k=1}^{q} d_{i k}\left(T_{*}\right) \Gamma_{i j} d_{j k}\left(T_{*}\right)^{*} ;
$$

(iii) there exist a Hilbert space $K$ and a unitary operator

$$
U=\left(\begin{array}{ll}
a & b \\
c & d
\end{array}\right) \in B\left(K^{p} \oplus E, K^{q} \oplus E_{*}\right)
$$

such that if $F(z)=d+c\left(1_{K^{p}}-d_{K}(z) a\right)^{-1} d_{K}(z) b$, then $X P_{M}=P_{M_{*}} T_{F}$.

Proof. The equivalence of conditions (i) and (iii) follows from Theorem 2.5.

To prove the implication (ii) $\Rightarrow$ (iii), suppose that $\Gamma \in B\left(M_{*}\right)^{p}$ is a positive operator as in condition (ii). Define $K_{0}=M_{*}^{p}$ and $L=\Gamma^{1 / 2}$. Write $L=\left(L_{1}, \ldots, L_{p}\right)^{t} \in B\left(K_{0}, M_{*}^{p}\right)$ as a column operator. Then $\Gamma_{i j}=L_{i} L_{j}^{*}$ for $1 \leq i, j \leq p$ and, using Proposition 1.3, we obtain the identity

$$
\left\|P_{E_{*}} h\right\|^{2}-\left\|P_{E} X^{*} h\right\|^{2}=\left\|\left(L_{j}^{*} h\right)_{j=1}^{p}\right\|^{2}-\left\|\left(\sum_{j=1}^{p} L_{j}^{*} d_{j k}\left(T_{*}\right)^{*} h\right)_{k=1}^{q}\right\|^{2}
$$

for all $h \in M_{*}$. Hence the map

$$
\left(\sum_{j=1}^{p} L_{j}^{*} d_{j k}\left(T_{*}\right)^{*} h\right)_{k=1}^{q} \oplus\left(P_{E_{*}} h\right) \mapsto\left(L_{j}^{*} h\right)_{j=1}^{p} \oplus\left(P_{E} X^{*} h\right)
$$

defines an isometry $V$ from the linear subspace of $K_{0}^{q} \oplus E_{*}$ consisting of the vectors on the left-hand side into the space $K_{0}^{p} \oplus E$. Choose a Hilbert space $K \supset K_{0}$ and a unitary operator $U \in B\left(K^{p} \oplus E, K^{q} \oplus E_{*}\right)$ such that $U^{*}$ extends $V$. Let $a, b, c, d$ be the coefficients of $U$ (as in condition (iii) of Proposition 3.7). Defining $\Phi_{j}=L_{j} P_{K_{0}} \in B\left(K, M_{*}\right.$ ) for $j=1, \ldots, p$ we obtain that

$$
\begin{aligned}
& a^{*}\left(\sum_{j=1}^{p} \Phi_{j}^{*} d_{j k}\left(T_{*}\right)^{*} h\right)_{k=1}^{q}+c^{*} P_{E_{*}} h=\left(\Phi_{j}^{*} h\right)_{j=1}^{p}, \\
& b^{*}\left(\sum_{j=1}^{p} \Phi_{j}^{*} d_{j k}\left(T_{*}\right)^{*} h\right)_{k=1}^{q}+d^{*} P_{E_{*}} h=P_{E} X^{*} h
\end{aligned}
$$

for all $h \in M_{*}$. According to Lemma 3.2 the unitary operator $U$ induces a contraction $\Omega=\left(\Omega_{1}, \ldots, \Omega_{p}\right): K^{p} \rightarrow \mathcal{H} \otimes E_{*}$ uniquely determined by

$$
\Omega^{*}\left(C_{z} \otimes x\right)=\left(1_{K^{p}}-a^{*} d_{K}(z)^{*}\right)^{-1} c^{*} x \quad\left(z \in D, x \in E_{*}\right) .
$$

It follows that

$$
\begin{aligned}
& a^{*} d_{K}(z)^{*} \Omega^{*}\left(C_{z} \otimes x\right)+c^{*} x=\Omega^{*}\left(C_{z} \otimes x\right), \\
& b^{*} d_{K}(z)^{*} \Omega^{*}\left(C_{z} \otimes x\right)+d^{*} x=P_{E} T_{F_{U}}^{*}\left(C_{z} \otimes x\right)
\end{aligned}
$$


for $x \in E_{*}$ and $z \in D$. Since the vectors $C_{z} \otimes x$ span $\mathcal{H} \otimes E_{*}$ topologically, the identity

$$
d_{K}(z)^{*} \Omega^{*}\left(C_{z} \otimes x\right)=\left(\sum_{j=1}^{p} \Omega_{j}^{*}\left(d_{j k}(Z)^{*} \otimes 1_{E_{*}}\right)\left(C_{z} \otimes x\right)\right)_{k=1}^{q}
$$

implies that

$$
\begin{aligned}
& a^{*}\left(\sum_{j=1}^{p} \Omega_{j}^{*}\left(d_{j k}(Z)^{*} \otimes 1_{E_{*}}\right) h\right)_{k=1}^{q}+c^{*} P_{E_{*}} h=\left(\Omega_{j}^{*} h\right)_{j=1}^{p}, \\
& b^{*}\left(\sum_{j=1}^{p} \Omega_{j}^{*}\left(d_{j k}(Z)^{*} \otimes 1_{E_{*}}\right) h\right)_{k=1}^{q}+d^{*} P_{E_{*}} h=P_{E} T_{F_{U}}^{*} h
\end{aligned}
$$

for all $h \in \mathcal{H} \otimes E_{*}$. Define $\Phi_{j}^{\prime}=P_{M_{*}} \Omega_{j} \in B\left(K, M_{*}\right)$ and $\Psi_{j}=\Phi_{j}-\Phi_{j}^{\prime} \in B\left(K, M_{*}\right)$ for $j=1, \ldots, p$. Then $\left(\Phi_{j}^{\prime}\right)^{*} h=\Omega_{j}^{*} h$ for $h \in M_{*}$. Taking the difference of the representations of $\Phi_{j}^{*} h$ and $\Omega_{j}^{*} h$ obtained above, and by using the fact that

$$
\left(d_{j k}(Z)^{*} \otimes 1_{E_{*}}\right) \mid M_{*}=d_{j k}\left(T_{*}\right)^{*},
$$

we conclude that

$$
a^{*}\left(\sum_{j=1}^{p} \Psi_{j}^{*} d_{j k}\left(T_{*}\right)^{*} h\right)_{k=1}^{q}=\left(\Psi_{j}^{*} h\right)_{j=1}^{p} \quad\left(h \in M_{*}\right) .
$$

Since $a^{*}$ is a contraction, we find that

$$
\sum_{j=1}^{p}\left\|\Psi_{j}^{*} h\right\|^{2} \leq \sum_{k=1}^{q}\left\|\sum_{j=1}^{p} \Psi_{j}^{*} d_{j k}\left(T_{*}\right)^{*} h\right\|^{2} \quad\left(h \in M_{*}\right) .
$$

Hence $\Delta=\left(\Psi_{i} \Psi_{j}^{*}\right)_{i, j} \in B\left(M_{*}^{p}\right)$ is a positive operator such that

$$
\sum_{j=1}^{p} \Delta_{j j}-\sum_{i, j=1}^{p} \sum_{k=1}^{q} d_{i k}\left(T_{*}\right) \Delta_{i j} d_{j k}\left(T_{*}\right)^{*} \leq 0 .
$$

By Proposition 3.6 the positivity of the operator $\Delta$ implies the positivity of the induced sesquilinear form $\tau \Delta=M_{0} \times M_{0} \rightarrow \mathbb{C}$. Using the definition of $\tau \Delta$ one obtains that $\tau \Delta$ is negative. Hence $\tau \Delta=0$, and again by Proposition 3.6, it follows that $\Delta=0$. But then

$$
\Phi_{j}^{*}=\Phi_{j}^{*}=\Omega_{j}^{*} \mid M_{*} \quad(1 \leq j \leq p) .
$$

Comparing the above representations of $P_{E} X^{*} h$ and $P_{E} T_{F_{U}}^{*} h$, we obtain that

$$
P_{E} X^{*} h=P_{E} T_{F_{U}}^{*} h \quad\left(h \in M_{*}\right) .
$$

Then, for any constant function $x \in E \subset \mathcal{H} \otimes E$ and any multiindex $\alpha \in \mathbb{N}^{n}$, we have

$$
\begin{aligned}
& \left\langle X^{*} h,\left(Z^{\alpha} \otimes 1_{E}\right) x\right\rangle=\left\langle T^{* \alpha} X^{*} h, x\right\rangle=\left\langle X^{*} T_{*}^{* \alpha} h, x\right\rangle \\
& =\left\langle T_{F_{U}}^{*} T_{*}^{* \alpha} h, x\right\rangle=\left\langle Z^{* \alpha} \otimes 1_{E} T_{F_{U}}^{*} h, x\right\rangle=\left\langle T_{F_{U}}^{*} h,\left(Z^{\alpha} \otimes 1_{E}\right) x\right\rangle .
\end{aligned}
$$

Since $\mathcal{H} \otimes E$ is spanned topologically by the elements of the form $z^{\alpha} \otimes x\left(\alpha \in \mathbb{N}^{n}\right.$, $x \in E$ ), we find that $X^{*} h=T_{F_{U}}^{*} h$ for each $h \in M_{*}$. Thus the proof of the implication (ii) $\Rightarrow$ (iii) is complete.

We complete the proof of Theorem 3.7 by showing that (iii) $\Rightarrow$ (ii). Suppose that $F=F_{U}$ for a unitary matrix operator as in condition (iii). The hypothesis that $X P_{M}=$ $P_{M_{*}} T_{F}$ is easily seen to be equivalent to the condition that $T_{F}^{*} M_{*} \subset M$ and $X^{*}=T_{F}^{*} \mid M_{*}$. 
Exactly as in the previous part of the proof, we apply Lemma 3.2 to the first column of the matrix operator $U$ to define a contraction $\Omega=\left(\Omega_{1}, \ldots, \Omega_{p}\right): K^{p} \rightarrow \mathcal{H} \otimes E_{*}$. The same arguments as in the proof of the implication (ii) $\Rightarrow$ (iii) show that

$$
U^{*}\left(\left(\sum_{j=1}^{p} \Omega_{j}^{*}\left(d_{j k}(Z)^{*} \otimes 1_{E_{*}}\right) h\right)_{k=1}^{q} \oplus\left(P_{E_{*}} h\right)\right)=\left(\Omega^{*} h\right) \oplus P_{E} T_{F}^{*} h
$$

for each $h \in \mathcal{H} \otimes E_{*}$. Using Proposition 1.3 and the fact that $U^{*}$ is isometric, we see that

$$
\begin{aligned}
\left\langle\frac{1}{C}\left(M_{T_{*}}\right)\left(1-X X^{*}\right) h, h\right\rangle & =\left\|P_{E_{*}} h\right\|^{2}-\left\|P_{E} X^{*} h\right\|^{2} \\
& =\left\|\Omega^{*} h\right\|^{2}-\left\|\left(\sum_{j=1}^{p} \Omega_{j}^{*} d_{j k}\left(T_{*}\right)^{*} h\right)_{k=1}^{q}\right\|^{2}
\end{aligned}
$$

for all $h \in M_{*}$. Now it is elementary to check that condition (ii) holds with

$$
\Gamma=\left(P_{M_{*}} \Omega_{i} \Omega_{j}^{*} \mid M_{*}\right)_{i j} \in B\left(M_{*}^{p}\right) .
$$

Thus the proof of Theorem 3.7 is complete.

REMARK 3.8. The hypothesis that $M_{0} \subset M_{*}$ is dense is only used to prove that (ii) implies (iii) in Theorem 3.7. We do not know whether this implication remains true without this hypothesis. For $p=1$, no condition on $M_{0}$ is needed. Indeed, in this case, one obtains exactly as in the above proof a positive operator $\Delta \in B\left(M_{*}\right)$ such that

$$
\Delta-\sum_{k=1}^{q} d_{k}\left(T_{*}\right) \Delta d_{k}\left(T_{*}\right)^{*} \leq 0 .
$$

To prove that (ii) implies (iii) one has to show that $\Delta=0$. By a well-known argument (see the proof of Theorem 3.10 in [9] it suffices to check that the row contraction $d\left(T_{*}\right) \in$ $B\left(M_{*}\right)^{q}$ is pure, i.e., that

$$
\text { SOT- } \lim _{N \rightarrow \infty} \Sigma_{d\left(T_{*}\right)}^{N}(1)=0
$$

where $\Sigma_{d\left(T_{*}\right)}: B\left(M_{*}\right) \rightarrow B\left(M_{*}\right), X \mapsto \sum_{k=1}^{q} d_{k}\left(T_{*}\right) X d_{k}\left(T_{*}\right)^{*}$. But this follows easily from the observation that

$$
\Sigma_{d\left(Z \otimes 1_{E_{*}}\right)}^{N}(1)\left(C_{w} \otimes x\right)=\left(\sum_{k=1}^{q} \overline{d_{k}(w)} d_{k}\left(Z \otimes 1_{E_{*}}\right)\right)^{N}\left(C_{w} \otimes x\right) \stackrel{N}{\rightarrow} 0
$$

for $x \in E_{*}$ and $w \in D$.

Our final aim in this section is to describe typical cases in which the set

$$
M_{0}=\left\{x \in M_{*} ; \sum_{k=0}^{\infty}\left\|e_{k}\left(T_{*}\right)^{*} x\right\|^{2}<\infty\right\}
$$

is automatically dense in $M_{*}$.

LEMma 3.9. Suppose that $\sigma\left(T_{*}\right) \subset D$. Then the series $\sum_{k=0}^{\infty} e_{k}\left(T_{*}\right) e_{k}\left(T_{*}\right)^{*}$ is norm convergent.

Proof. The condition that $\sigma\left(T_{*}\right) \subset D$ implies that $\sigma\left(M_{T_{*}}\right)=\sigma\left(T_{*}\right) \times \sigma\left(T_{*}^{*}\right) \subset \Delta$. Since the series $C(z, w)=\sum_{k=0}^{\infty} e_{k}(z) \tilde{e}_{k}(w)$ converges uniformly on all compact subsets of $\Delta$, we obtain the representation $C\left(T_{*}, T_{*}^{*}\right)=\sum_{k=0}^{\infty} e_{k}\left(T_{*}\right) e_{k}\left(T_{*}\right)^{*}$.

A similar situation occurs when $M_{*}$ is finite-dimensional. 
LEMMA 3.10. Let $M_{*} \subset \mathcal{H} \otimes E_{*}$ be a finite-dimensional $*$-invariant subspace. Then the series $\sum_{k=0}^{\infty} e_{k}\left(T_{*}\right) e_{k}\left(T_{*}\right)^{*}$ is norm convergent.

Proof. Note that the set

$$
L=\left\{A \in B\left(M_{*}\right) ; \sum_{k=0}^{\infty} e_{k}\left(T_{*}\right) A e_{k}\left(T_{*}\right)^{*} \text { converges }\right\}
$$

is a linear subspace of the finite-dimensional normed space $B\left(M_{*}\right)$. Because of

$$
\text { SOT- } \sum_{k=0}^{\infty} e_{k}\left(Z \otimes 1_{E_{*}}\right) P_{E_{*}} e_{k}\left(Z \otimes 1_{E_{*}}\right)^{*}=\mathrm{SOT}-\sum_{k=0}^{\infty}\left(\left\langle\cdot, e_{k}\right\rangle e_{k}\right) \otimes 1_{E_{*}}=1_{\mathcal{H} \otimes \mathcal{E}_{*}}
$$

it suffices to show that the operator $C_{j}=P_{M_{*}} e_{j}\left(Z \otimes 1_{E_{*}}\right) P_{E_{*}} e_{j}\left(Z \otimes 1_{E_{*}}\right)^{*} \mid M_{*}$ belongs to $L$ for each fixed natural number $j$. But this easily follows from the identity

$$
e_{k}\left(T_{*}\right) C_{j} e_{k}\left(T_{*}\right)^{*}=e_{j}\left(T_{*}\right) C_{k} e_{j}\left(T_{*}\right)^{*}
$$

valid for all $k \geq 0$.

By applying the previous lemma to the scalar case $E_{*}=\mathbb{C}$, one finds that the series $\sum_{k=0}^{\infty} e_{k}\left(T_{*}\right) e_{k}\left(T_{*}\right)^{*}$ is also norm convergent on any *- invariant subspace $M \otimes E_{*}$ of $\mathcal{H} \otimes E_{*}$ determined by a finite-dimensional $Z^{*}$-invariant subspace $M \subset \mathcal{H}$.

REMARK 3.11. (a) If $M_{*}$ is the closed linear span of an arbitrary family of $*$-invariant subspaces $M_{i} \subset \mathcal{H} \otimes E_{*}(i \in I)$ such that, for each $i$, the subset

$$
\left\{x \in M_{i} ; \sum_{k=0}^{\infty}\left\|e_{k}\left(P_{M_{i}}\left(Z \otimes 1_{E_{*}}\right) \mid M_{i}\right)^{*} x\right\|^{2}<\infty\right\} \subset M_{i}
$$

is dense, then $M_{0} \subset M_{*}$ is dense. In particular, if

$$
M_{*}=\bigvee\left(\operatorname{Ker}\left(\bar{\lambda}-\mathrm{T}_{*}^{*}\right)^{\alpha} ; \lambda \in \mathrm{D} \text { and } \alpha \in \mathbb{N}^{\mathrm{n}}\right)
$$

then Lemma 3.9 implies that $M_{0} \subset M_{*}$ is dense.

(b) On the other hand, there are elementary examples showing that in general the set $M_{0}$ can be quite small. Let $\mathcal{H}$ be the Hardy space on the unit disc $\mathbb{D}$ in $\mathbb{C}$ and choose $\left(e_{k}\right)_{k \geq 0}=\left(z^{k}\right)_{k \geq 0}$. Each $C .0^{-}$-contraction $T \in B(H)$ on an arbitrary Hilbert space $H$ is, up to unitary equivalence, the compression of $Z \otimes 1_{E_{*}} \in B\left(\mathcal{H} \otimes E_{*}\right)$ to a $*$-invariant subspace, where $E_{*}$ is a suitably chosen Hilbert space (Theorem VI.2.3 in [31]). In particular, this applies to the adjoint $T=M_{z}^{*} \in B\left(L_{a}^{2}(\mathbb{D})\right)$ of the Bergman shift defined on the Hilbert space $L_{a}^{2}(\mathbb{D})$ of all square integrable analytic functions on $\mathbb{D}$. But an elementary exercise shows that

$$
\left\{f \in L_{a}^{2}(\mathbb{D}) ; \sum_{k=0}^{\infty}\left\|e_{k}(T)^{*} f\right\|^{2}<\infty\right\}=\left\{f \in L_{a}^{2}(\mathbb{D}) ; \sum_{k=0}^{\infty}\left\|z^{k} f\right\|^{2}<\infty\right\}=\{0\} .
$$

4. Interpolation for Schur class functions. The above results can be used to solve interpolation problems for Schur class functions. For simplicity, we only treat the scalarvalued case, that is, in the following we make the assumption that $E=E_{*}=\mathbb{C}$.

Let $\mathcal{H}$ be a functional Hilbert space consisting of analytic functions on an open set

$$
D=\{z \in W ;\|d(z)\|<1\}
$$


in $\mathbb{C}^{n}$ such that $\mathcal{H}$ and $D$ satisfy all hypotheses described at the beginning of Section 3. We fix a finite subset $S \subset D$ and suppose that, for each $s \in S$, a finite set $A_{s} \subset \mathbb{N}^{n}$ is given with the property that, for each $\alpha \in A_{s}$, we have

$$
\left\{\gamma \in \mathbb{N}^{n} ; \gamma \leq \alpha\right\} \subset A_{s}
$$

Here the order relation $\gamma \leq \alpha$ is defined componentwise. For each $s \in S$, let $\left(c_{s, \alpha}\right)_{\alpha \in A_{s}}$ be a fixed family of complex numbers. Our aim in the following is to find conditions that characterize the existence of functions $f \in \mathcal{S}_{d}$ in the Schur class such that

$$
f^{(\alpha)}(s) / \alpha !=c_{s, \alpha} \quad\left(s \in S, \alpha \in A_{s}\right) .
$$

Since every Schur class function $f \in \mathcal{S}_{d}$ is a multiplier of $\mathcal{H}$ with $\left\|T_{f}\right\| \leq 1$, we find at least sufficient conditions for the existence of interpolating functions with multiplier norm or supremum norm bounded by one.

Since by the closed graph theorem the inclusion mapping $\mathcal{H} \subset \mathcal{O}(D)$ is continuous, for each point $w \in D$ and each multiindex $\alpha \in \mathbb{N}^{n}$, there is a unique function $C_{w}^{\alpha} \in \mathcal{H}$ with the property that

$$
\left\langle f, C_{w}^{\alpha}\right\rangle=f^{(\alpha)}(w) / \alpha ! \quad(f \in \mathcal{H}) .
$$

To simplify the notation we write $f_{(\alpha)}(w)=f^{(\alpha)}(w) / \alpha$ ! for $f \in \mathcal{O}(D), w \in D$ and $\alpha \in \mathbb{N}^{n}$. Let $\left(e_{k}\right)_{k \in \mathbb{N}}$ be an orthonormal basis of $\mathcal{H}$. Then

$$
C(z, w)=\sum_{k=0}^{\infty} e_{k}(z) \tilde{e}_{k}(w)
$$

where the series converges uniformly on compact subsets of $\Delta=\{(z, \bar{w}) ; z, w \in D\}$. Hence, for $\alpha \in \mathbb{N}^{n}$ and $z, w \in D$, we find that

$$
\left(\partial_{w}^{\alpha} C\right)(z, \bar{w})=\sum_{k=0}^{\infty}\left(\partial^{\alpha} e_{k}\right)^{\sim}(\bar{w}) e_{k}(z)=\sum_{k=0}^{\infty} \overline{\left(\partial^{\alpha} e_{k}\right)(w)} e_{k}(z)=\alpha ! C_{w}^{\alpha}(z) .
$$

Since, for $z, w \in D$, the identity

$$
C_{z}(w)=C(w, \bar{z})=\overline{C(z, \bar{w})}=C(z, \cdot)^{\sim}(w)
$$

holds, it follows that

$$
\left(\overline{\left.\partial^{\alpha} C_{z}\right)(w)}=\left(\partial^{\alpha} C(z, \cdot)\right)(\bar{w})=\alpha ! C_{w}^{\alpha}(z) .\right.
$$

Lemma 4.1. Let $f \in \mathcal{O}(D)$ be a multiplier of $\mathcal{H}$. Then the identity

$$
T_{f}^{*} C_{w}^{\alpha}=\sum_{0 \leq \gamma \leq \alpha} \overline{f_{(\alpha-\gamma)}(w)} C_{w}^{\gamma}
$$

holds for all points $w \in D$ and each multiindex $\alpha \in \mathbb{N}^{n}$.

Proof. It suffices to observe that

$$
T_{f}^{*} C_{w}^{\alpha}(z)=\left\langle C_{w}^{\alpha}, T_{f} C_{z}\right\rangle=\overline{\left(f C_{z}\right)_{(\alpha)}(w)}=\sum_{0 \leq \gamma \leq \alpha} \overline{f_{(\alpha-\gamma)}(w)} C_{w}^{\gamma}(z)
$$

for all $z, w \in D$ and $\alpha \in \mathbb{N}^{n}$.

By Lemma 4.1 the subspace

$$
M=M_{*}=\operatorname{LH}\left\{C_{s}^{\alpha} ; s \in S \text { and } \alpha \in A_{s}\right\}
$$


is invariant for the commuting tuple $Z^{*} \in B(\mathcal{H})^{n}$. As before, we denote by $T \in B(M)^{n}$ the compression of $Z$ to $M$. Since the generating vectors $C_{s}^{\alpha}$ for $M$ are linearly independent, there is a unique operator $X \in B(M)$ such that

$$
X^{*} C_{s}^{\alpha}=\sum_{0 \leq \gamma \leq \alpha} \bar{c}_{s, \alpha-\gamma} C_{s}^{\gamma}
$$

for all $s \in S$ and $\alpha \in A_{s}$. The existence of a Schur class function $f \in \mathcal{S}_{d}$ with

$$
f_{(\alpha)}(s)=c_{s, \alpha} \quad\left(s \in S \text { and } \alpha \in A_{s}\right)
$$

is equivalent to the existence of a function $f \in \mathcal{S}_{d}$ with $T_{f}^{*} M \subset M$ and $T_{f}^{*} \mid M=X^{*}$. It is elementary to check that $X T_{j}=T_{j} X$ for $j=1, \ldots, n$. Hence Theorem 3.7 can be applied to characterize the solvability of the above interpolation problem.

Our aim is to prove this interpolation result in a form which directly generalizes the classical theorem known for the case of the unit disc. For this aim, let $P$ denote the orthogonal projection from $\mathcal{H}$ onto the constant functions. Then we obtain that

$$
P C_{s}^{\alpha}=\left\langle C_{s}^{\alpha}, 1\right\rangle=\delta_{\alpha, 0} 1 \quad\left(s \in S, \alpha \in A_{s}\right) .
$$

Using Lemma 4.1 and the results from Section 1, one easily finds that

$$
\left\langle\frac{1}{C}\left(M_{T}\right)\left(1-X X^{*}\right) C_{t}^{\beta}, C_{s}^{\alpha}\right\rangle=\delta_{(\alpha, \beta),(0,0)}-c_{s, \alpha} \bar{c}_{t, \beta}
$$

for all $s, t \in S$ and $\alpha \in A_{s}, \beta \in A_{t}$. Our result is formulated in terms of a scalar matrix $G=\left(G_{\rho \sigma}\right)_{\rho, \sigma \in \Lambda}$, where $\Lambda$ is the index set

$$
\Lambda=\left\{\rho=(j, s, \alpha) ; j=1, \ldots, p \text { and } s \in S, \alpha \in A_{s}\right\} .
$$

TheOREm 4.2 (Carathéodory-Fejér problem). For $S \subset D$ finite and finite families $\left(c_{s, \alpha}\right)_{\alpha \in A_{s}}(s \in S)$ as above, the following are equivalent:

(i) there is a function $f \in \mathcal{S}_{d}$ with $f^{(\alpha)}(s) / \alpha$ ! $=c_{s, \alpha}$ for all $s \in S$ and $\alpha \in A_{s}$;

(ii) there is a positive semidefinite matrix $G=\left(G_{\rho \sigma}\right)_{\rho, \sigma \in \Lambda}$ of complex numbers such that the equations

$$
\begin{aligned}
\delta_{(\alpha, \beta),(0,0)}-c_{s, \alpha} \bar{c}_{t, \beta}= & \sum_{j=1}^{p} G_{(j, s, \alpha)(j, t, \beta)} \\
& -\sum_{\substack{0 \leq \delta \leq \alpha \\
0 \leq \lambda \leq \beta}} \sum_{i, j=1}^{p}\left(\sum_{k=1}^{q}\left(d_{i k}\right)_{(\alpha-\delta)}(s) \overline{\left(d_{j k}\right)_{(\beta-\lambda)}(t)}\right) G_{(i, s, \delta)(j, t, \lambda)}
\end{aligned}
$$

hold for all $s, t \in S$ and $\alpha \in A_{s}, \beta \in A_{t}$.

Proof. By Theorem 3.7 the validity of condition (i) is equivalent to the existence of a positive operator $\Gamma=\left(\Gamma_{i j}\right) \in B\left(M^{p}\right)$ such that

$$
\frac{1}{C}\left(M_{T}\right)\left(1-X X^{*}\right)=\sum_{j=1}^{p} \Gamma_{j j}-\sum_{i, j=1}^{p} \sum_{k=1}^{q} d_{i k}(T) \Gamma_{i j} d_{j k}(T)^{*} .
$$

Each coefficient $\Gamma_{i j} \in B(M)$ has a representation of the form

$$
\Gamma_{i j} C_{t}^{\beta}=\sum_{u \in S, \nu \in A_{u}} \Gamma_{u, \nu}^{t \beta}(i, j) C_{u}^{\nu} \quad\left(t \in S, \beta \in A_{t}\right) .
$$


By applying both sides of the first equation to the vector $C_{t}^{\beta}$, and then forming the inner product with $C_{s}^{\alpha}$, one obtains the identity

$$
\begin{aligned}
\delta_{(\alpha, \beta),(0,0)}-c_{s, \alpha} \bar{c}_{t, \beta}= & \sum_{u \in S, \nu \in A_{u}}\left[\sum_{j=1}^{p} \Gamma_{u \nu}^{t \beta}(j, j) C_{(\alpha, \nu)}(s, \bar{u})\right. \\
& \left.-\sum_{i, j=1}^{p} \sum_{\substack{k=1 \\
k=1}} \sum_{\substack{0 \leq \delta \leq \alpha \\
0 \leq \lambda \leq \beta}}\left(d_{i k}\right)_{(\alpha-\delta)}(s) \overline{\left(d_{j k}\right)_{(\beta-\lambda)}(t)} \Gamma_{u \nu}^{t \lambda}(i, j) C_{(\delta, \nu)}(s, \bar{u})\right]
\end{aligned}
$$

for all $s, t \in S$ and $\alpha \in A_{s}, \beta \in A_{t}$. The scalar matrix $G=\left(G_{\rho \sigma}\right)_{\rho, \sigma \in \Lambda}$ with coefficients defined by

$$
G_{(i, s, \alpha)(j, t, \beta)}=\sum_{u \in S, \nu \in A_{u}} \Gamma_{u \nu}^{t \beta}(i, j) C_{(\alpha, \nu)}(s, \bar{u})
$$

clearly satisfies condition (ii). The positivity of the matrix operator $\Gamma=\left(\Gamma_{i j}\right) \in B\left(M^{p}\right)$ is equivalent to the positive semidefiniteness of the matrix $G$. To check this, it suffices to observe that, for every vector $m=\left(m_{j}\right) \in M^{p}$ with $m_{j}=\sum_{t \in S, \beta \in A_{t}} v_{(j, t, \beta)} C_{t}^{\beta}$ for $i=1, \ldots, p$, the identity

$$
\sum_{i, j=1}^{p}\left\langle\Gamma_{i j} m_{j}, m_{i}\right\rangle=\sum_{\rho, \sigma \in \Lambda} G_{\sigma \rho} v_{\rho} \bar{v}_{\sigma}
$$

holds. Thus it is clear that condition (i) implies condition (ii).

If conversely, a positive semidefinite matrix $G$ as in condition (ii) is given, then the last equality can be used to define a positive matrix operator $\Gamma \in B\left(M^{p}\right)$. By reversing the above arguments one finds that $\Gamma$ satisfies the equation contained in condition (ii) of Theorem 3.7 if the operator $X$ is defined as above.

In the particular case that $A_{s}=\{0\}$ for each $s \in S$, the preceding result yields a solution of the Nevanlinna-Pick problem for Schur class functions. The same result and various similar characterizations, even for an arbitrary subset $S \subset D$, are contained in Theorem 2.5. As an example we state the result below, for which we also cite [5].

THEOREM 4.3. Let $S \subset D$ be an arbitrary subset, and let $\left(c_{s}\right)_{s \in S}$ be a family of complex numbers. Then there is a Schur class function $f \in \mathcal{S}_{d}$ with $f(s)=c_{s}$ for all $s \in S$ if and only if there is a positive definite function $\Gamma=\left(\Gamma_{i j}\right): S \times S \rightarrow B\left(\mathbb{C}^{p}\right) \cong \mathbb{C}^{p, p}$ such that

$$
1-c_{s} \bar{c}_{t}=\sum_{j=1}^{p} \Gamma_{j j}(t, s)-\sum_{i, j=1}^{p} \sum_{k=1}^{q} d_{i k}(t) \overline{d_{j k}(t)} \Gamma_{i j}(t, s)
$$

holds for all $s, t \in S$.

Proof. The assertion follows directly from the equivalence of conditions (i) and (ii)' in Theorem 2.5.

\section{References}

[1] J. Agler, On the representation of certain holomorphic functions defined on the polydisc, in: Topics in Operator Theory: Ernst D. Hellinger Memorial Volume, Operator Theory: Advances and Applications, 48, Birkhäuser, Basel, 1990, 47-66. 
[2] J. Agler and J.E. McCarthy, Nevanlinna-Pick interpolation on the bidisc, J. Reine Angew. Math. 506 (1999), 191-204.

[3] C.-G. Ambrozie, M. Engliš and V. Müller, Analytic models over general domains in $\mathbb{C}^{n}$, J. Operator Theory 47 (2002), 287-302.

[4] C.-G. Ambrozie and D. Timotin, On an intertwining lifting for certain reproducing kernel Hilbert spaces, Integral Equations Operator Theory 42 (2002), 373-384.

[5] C.-G. Ambrozie and D. Timotin, A von Neumann type inequality for certain domains in $\mathbb{C}^{n}$, Proc. Amer. Math. Soc., to appear.

[6] A. Arias and G. Popescu, Noncommutative interpolation and Poisson transforms, Israel J. Math. 115 (2000), 205-234.

[7] N. Aronszajn, Theory of reproducing kernels, Trans. Amer. Math. Soc. 68 (1950), 337-404.

[8] W. Arveson, Subalgebras of $C^{*}$-algebras III, Multivariable operator theory, Acta Math. 181 (1998), 159-228.

[9] W. Arveson, The curvature invariant of a Hilbert module over $\mathbb{C}\left[z_{1}, \ldots, z_{n}\right]$, J. Reine Angew. Math. 522 (2000), 173-236.

[10] C. Badea and G. Cassier, Constrained von Neumann inequalities, Adv. Math. 166 (2002), 260-297.

[11] J. A. Ball and V. Bolotnikov, Realization and interpolation for Schur-Agler-class functions on domains with matrix polynomial defining function in $\mathbb{C}^{n}$, preprint.

[12] J. A. Ball, W. S. Li, D. Timotin and T. T. Trent, A commutant lifting theorem on the polydisc, Indiana Univ. Math. J. 48 (1999), 653-675.

[13] J. A. Ball, T. T. Trent and V. Vinnikov, Interpolation and commutant lifting for multipliers on reproducing kernel Hilbert spaces, in: Operator Theory and Analysis, OT 122, Birkhäuser, Basel, 2001, 89-138.

[14] C. Barbian, Positivitätsbedingungen funktionaler Hilberträume und Anwendungen in der mehrdimensionalen Operatorentheorie. Diplomarbeit, Universität des Saarlandes, 2001.

[15] F. Beatrous and J. Burbea, Reproducing kernels and interpolation for holomorphic functions, in: Complex Analysis, Functional Analysis and App. Theory (Campinas, 1984), North-Holland Math. Studies 125 (1986), 25-46.

[16] K. R. Davidson and D. R. Pitts, Nevanlinna-Pick interpolation for non-commutative analytic Toeplitz algebras, Integral Equations Operator Theory 31 (1998), 321-337.

[17] J. Eschmeier, Tensor products and elementary operators, J. Reine Angew. Math. 390 (1988), 47-66.

[18] J. Eschmeier and M. Putinar, Spectral Decompositions and Analytic Sheaves, London Math. Soc. Monographs, 10, Clarendon Press, Oxford, 1996.

[19] J. Eschmeier and M. Putinar, Spherical contractions and interpolation problems on the unit ball, J. Reine Angew. Math. 542 (2002), 219-236.

[20] J. Eschmeier, L. Patton and M. Putinar, Carathéodory-Féjer interpolation on polydisks, Math. Res. Lett. 7 (2000), 25-34.

[21] C. Foias and A. E. Frazho, The commutant lifting approach to interpolation problems, Operator Theory: Advances and Appl., 44, Birkhäuser, Basel, 1990.

[22] L. K. Hua, Harmonic analysis of functions of several complex variables in the classical domains, Amer. Math. Soc., Providence, Rhode Island, 1963.

[23] V. L. Klee, Separation properties of convex cones, Proc. Amer. Math. Soc. 6 (1955), 313-318.

[24] A. Korányi and L. Pukanszky, Holomorphic functions with positive real part on polycylinders, Trans. Amer. Math. Soc. 108 (1963), 449-456. 
[25] G. Popescu, Isometric dilations for infinite sequences of noncommuting operators, Trans. Amer. Math. Soc. 316 (1989), 523-536.

[26] G. Popescu, On intertwining dilations for sequences of noncommuting operators, J. Math. Anal. Appl. 167 (1992), 382-402.

[27] G. Popescu, Interpolation problems in several variables, J. Math. Anal. App. 227 (1998), 227-250.

[28] G. Popescu, Commutant lifting, tensor algebras, and functional calculus, Proc. Edinb. Math. Soc. 44 (2001), 389-406.

[29] D. Sarason, Generalized interpolation in $H^{\infty}$, Trans. Amer. Math. Soc. 127 (1967), 179203.

[30] Z. Słodkowski and W. Żelazko, On joint spectra of commuting families of operators, Studia Math. 50 (1974), 127-148.

[31] B. Sz.-Nagy and C. Foias, Harmonic Analysis of Operators on Hilbert space, NorthHolland, Amsterdam, 1970.

[32] F.-H. Vasilescu, Analytic Functional Calculus and Spectral Decompositions, Editura Academiei, Bucureşti, and D. Reidel, Dordrecht, 1982. 